Pacific Journal of Mathematics

COORDINATES FOR TRIANGULAR OPERATOR ALGEBRAS. II

Scott Muhly, Kichi-Suke Salto and Baruch Sol 
Pacific Journal of Mathematics

COORDINATES FOR TRIANGULAR OPERATOR ALGEBRAS. II

Scott Muhly, Kichi-Suke Salto and Baruch Sol 


\title{
COORDINATES FOR TRIANGULAR OPERATOR ALGEBRAS II
}

\section{Paul S. Muhly, Kichi-Suke Saito and Baruch Solel Dedicated to the memory of Henry A. Dye}

\begin{abstract}
Let $M$ be a von Neumann algebra and let $A$ be a maximal abelian self-adjoint subalgebra (masa) of $M$. A subalgebra $\mathfrak{T}$ of $M$ is called triangular (with respect to $A$ ) if $\mathfrak{T} \cap \mathfrak{T}^{*}=A$, where $\mathfrak{T}^{*}$ denotes the collection of adjoints of the elements in $\mathfrak{T}$. If $\mathfrak{T}$ is not contained in any larger triangular subalgebra of $M$, then $\mathfrak{T}$ is called maximal triangular. If $A$ is a Cartan subalgebra, then $M$ may be realized as an algebra of matrices indexed by an equivalence relation on a standard Borel space and if $\mathfrak{T}$ is $\sigma$-weakly closed and maximal triangular, then $\mathfrak{T}$ may be realized as the collection of matrices supported on the graph of a partial order that totally orders each equivalence class. In this paper we will be concerned with the relation between the structure of these algebras and the theory of analytic operator algebras. It turns out that this relation is complex: it involves the cohomology of the equivalence relation, the order type of the partial order and the type of $M$.
\end{abstract}

The concept of a triangular algebra was introduced formally in 1959 by Kadison and Singer in their fundamental paper [KS] which launched the theory of non-self-adjoint operator algebras. Their objective was to develop a theory of operator algebras whose elements could simultaneously all be put into triangular form. It is an elementary exercise to see that when $M$ is the full algebra of $n$ by $n$ matrices and when $A$ is the subalgebra of diagonal matrices, a masa in $M$, then given a triangular subalgebra $\mathfrak{T}$ of $M$, it is possible to conjugate $\mathfrak{T}$ by a permutation matrix so that the matrices in $\mathfrak{T}$ are all upper triangular. Moreover, $\mathfrak{T}$ is maximal triangular if and only if $\mathfrak{T}$ is (unitarily equivalent) to the algebra of all upper triangular matrices. In [MSS] we began a study of triangular algebras where it is assumed that $A$ is a Cartan subalgebra of $M$ in the sense of Feldman and Moore [FM1]. (This means that there is a faithful normal expectation from $M$ onto $A$ and that the group of unitary operators in $M$ that normalizes $A$ generates $M$.) Feldman and Moore showed that if $A$ is a Cartan subalgebra of $M$, then there is a standard Borel measure space $(X, \mathscr{B}, \mu)$ and a Borel equivalence relation $R \subseteq X \times X$, with the property that 
each equivalence class is countable, such that the operators in $M$ may be represented as matrices indexed by $R$. (The product between matrices may have to be twisted by a certain 2-cocycle and so we write $M=M(R, s)$ where $s$ is the 2-cocycle.) In this representation, $A$ is realized as $L^{\infty}(\mu)$ represented by the bounded measurable functions on the diagonal $\Delta$. It is a consequence of our Spectral Theorem for Bimodules, Theorem 2.5 of [MSS], that if $\mathfrak{T}$ is a $\sigma$-weakly closed triangular subalgebra of $M$ with respect to $A$, then there is a Borel subset $P$ of $R$ such that $T$ consists of all operators whose matrices are supported on $P$ and, moreover, $P$ is a partial order, i.e., a transitive, reflexive and antisymmetric relation. In fact, ignoring details about null sets, there is a one to one correspondence between such Borel partial orders and $\sigma$-weakly closed triangular subalgebras of $M(R, s)$, and we write $\mathfrak{T}=\mathfrak{T}(P)$. It turns out that $\mathfrak{T}$ is maximal triangular, if and only if $P$ totally orders every equivalence class of $R$. Thus, in every basic respect, the theory we developed generalizes the theory of triangular subalgebras of the $n \times n$ matrices.

Actually, in [MSS], we worked with the slightly more tractable class of subdiagonal algebras, first introduced by Arveson in [A1]. As is shown in [MSS], every (maximal) subdiagonal algebra of $M$ containing $A$ is of the form $\mathfrak{T}(P)$ for a transitive reflexive relation $P$ satisfying $P \cup \theta(P)=R$, where $\theta(x, y)=(y, x)$. Thus, in effect, such algebras are algebras of block upper triangular matrices. These are our primary objects of study.

In this paper, our objective is to see how this theory is related to the theory of analytic operator algebras as developed in [LM]. Recall that if $\mathfrak{R}$ is a von Neumann algebra and if $\alpha=\left\{\alpha_{t}\right\}_{t \in \mathbf{R}}$ is a one parameter group of automorphisms of $\mathfrak{R}$, then the collection $H^{\infty}(\alpha)$, which is defined to be all those $a$ in $R$ such that the map $t \rightarrow \rho\left(\alpha_{t}(a)\right)$ lies in the classical Hardy space $H^{\infty}(\mathbf{R})$ for every normal state on $\Re$, is a $\sigma$ weakly closed subalgebra of $\mathfrak{R}$ called the algebra of analytic operators in $\Re$. We are interested in how analyticity considerations are reflected in $P, \mathfrak{T}(P)$ and $M(R, s)$. We will see that given $P$, there is a real-valued function $d$ on $R$ such that $P=\{(x, y) \in R \mid d(x, y) \geq 0\}$. Of course the characteristic function of $P$ will do, but there are other choices which are more natural from our perspective. This $d$ will be used to show that $\mathfrak{T}(P)$ may always be represented as the intersection of $M(R, s)$ with a nest algebra in a nontrivial way. It will be shown that $\mathfrak{T}(P)$ is an analytic operator algebra in $M(R, s)$ if and only if it is possible to choose $d$ to be a cocycle for $R$, and $\mathfrak{T}(P)$ is a nest subalgebra of 
$M(R, s)$ if and only if it is possible to choose $d$ to be a coboundary. We will show, too, that if $\mathfrak{T}(P)$ is an analytic operator algebra in $M(R, s)$ for every $P$, then for each $P, \mathfrak{T}(P)$ is a nest subalgebra of $M(R, s)$. Moreover, assuming $M(R, s)$ is hyperfinite, this happens if and only if $M(R, s)$ is type $I$. In fact, if $M(R, s)$ is type $I$, then every $\mathfrak{T}(P)$ is a nest subalgebra of $M(R, s)$. In a sense, this is a generalization of the assertion that a $\sigma$-weakly closed, maximal triangular algebra in the full algebra of operators on Hilbert space is hyper-reducible and therefore a nest algebra [A3]. When we began our study of algebras of the form $\mathfrak{T}(P)$, we had hoped that they all would be analytic subalgebras of their enveloping von Neumann algebras. We wanted this in order to be able to make use of the invariant subspace theory developed in [LM] and elsewhere. Of course, this hope is now wrong. There must be $P$ 's for which $\mathfrak{T}(P)$ is not analytic. We will construct and examine some explicit examples. We will show that if $\mathfrak{T}(P)$ equals $H^{\infty}(\alpha)$ for a periodic action $\alpha$ on $M(R, s)$, then necessarily, $M(R, s)$ is hyperfinite and $\mathfrak{T}(P)$ decomposes as the direct sum of an analytic crossed product, determined by a Borel automorphism of a measure space, and a nest subalgebra of $M\left(R^{\prime}, s\right)$ where $R^{\prime}$ is a "corner" of $R$. We will show, too, that if $\mathfrak{T}(P)$ is analytic, then necessarily the order type of each $R$-equivalence class is very restricted.

The next section is devoted to some preliminary material taken from [FM1, 2] and from [MSS]. Section 3 is concerned with how our algebras are related to analytic operator algebras. We examine in particular what happens when $M$ is type $I$, and we draw some interesting conclusions about the relation between partial orders and the cohomology of $R$. Section 4 is devoted to the study of periodic flows. Here we give a first example where one of our algebras is not an analytic subalgebra of $M(R, s)$. Finally, in $\S 5$, we construct and analyze some other explicit examples of sets $P$ such that $\mathfrak{T}(P)$ is not an analytic subalgebra of $M(R, s)$.

Throughout this paper, all Hilbert spaces will be complex and separable. If $\mathscr{H}$ is a Hilbert space, then the algebra of all bounded linear operators on $\mathscr{H}$ will be denoted $\mathscr{L}(\mathscr{H})$. All measure spaces are standard Borel spaces.

2. Preliminaries. We recall here certain terminology, notation, and facts from the Feldman-Moore theory [FM1, 2] and from our paper [MSS]. 
Throughout, $X$ will denote a standard Borel space and $\mathfrak{B}$ will denote the underlying $\sigma$-field, called the $\sigma$-field of Borel sets in $X$. An equivalence relation $R \subseteq X \times X$ is called standard if it is simply a Borel subset relative to the product $\sigma$-field. If $R$ is an equivalence relation on $X$ and if $B \subseteq X$, we write $R(B)=\{y \mid(x, y) \in R$ for some $x \in B\}$; we write $R(x)$ for $R(\{x\}), x \in X$. We call $R$ countable (finite) if $R(x)$ is countable (finite) for every $x \in X$. Throughout, our equivalence relations will be standard and countable. If $R$ is such an equivalence relation, the product $\sigma$-field $\mathfrak{B} \times \mathfrak{B}$ restricted to $R$ will be denoted $\mathscr{C}$. The functions $\pi_{l}$ and $\pi_{r}$ mapping $R$ into $X$ according to the formulas $\pi_{l}(x, y)=x$ and $\pi_{r}(x, y)=y$ are called the left and right projections, respectively. Also, the map $\theta: R \rightarrow R$ defined above by the formula $\theta(x, y)=(y, x)$ plays an important role for us; it is called inversion.

A $\sigma$-finite measure $\mu$ on $(X, \mathfrak{B})$ is called quasi-invariant relative to the (standard, countable) equivalence relation $R$ if $\mu(R(A))=0$ for all sets $A$ such that $\mu(A)=0$. (Note that since $\pi_{l}$ and $\pi_{r}$ are countableto-one, they map sets in $\mathscr{C}$ to sets in $\mathfrak{B}$; consequently, $R(A) \in \mathfrak{B}$ for all $A \in \mathfrak{B}$.) We note that by Theorem 1 of [FM1], given an equivalence relation $R$ of the type we are considering, there is a countable group $G$ acting on $X$ by Borel automorphisms such that $R=R_{G}$ := $\{(x, g \cdot x) \mid x \in X, g \in G\}$. Observe that a measure $\mu$ on $X$ is quasiinvariant under $R_{G}$ if and only if $\mu$ is quasi-invariant under the action of $G$ in the usual sense.

A quasi-invariant measure $\mu$ on $X$ induces two measures $\nu_{l}$ and $\nu_{r}$ on $(R, \mathscr{C})$ via the formulas

$$
\nu_{l}(C)=\int\left|\pi_{l}^{-1}(x) \cap C\right| d \mu(x)
$$

and

$$
\nu_{r}(C)=\int\left|\pi_{r}^{-1}(x) \cap C\right| d \mu(x),
$$

where $C \in \mathscr{C}$ and where, for any set $S,|S|$ denotes the cardinality of $S$. The measures $\nu_{l}$ and $\nu_{r}$ are called the left and right counting measures on $\mathscr{C}$ determined by $\mu$. (Note that the assumption that $R$ is countable and standard implies that the functions $\left|\pi_{l}^{-1}(x) \cap C\right|$ and $\left|\pi_{r}^{-1}(x) \cap C\right|$ are Borel functions on $X$ for each $C \in \mathscr{C}$.) The quasi-invariance of $\mu$ is equivalent to the assertion that $\nu_{l}$ and $\nu_{r}$ are mutually absolutely continuous (cf. Proposition 2.1 and Theorem 2 of [FM1]). The Radon-Nikodym derivative $d \nu_{l} / d \nu_{r}$ will be denoted $D$. We will say that $\mu$ is invariant if $D \equiv 1$ a.e. Observe that when $R=R_{G}$, invariance in this sense is the same as the usual term. 
Let $A$ and $B$ be Borel subsets of $X$ and let $\varphi$ be a Borel isomorphism mapping $A$ onto $B$. We call $\varphi$ a partial Borel isomorphism on $X$; we call $A$ the domain of $\varphi$ and write $A=d(\varphi)$, and we call $B$ the range of $\varphi$ and write $B=r(\varphi)$. Evidently, $\varphi \circ \varphi^{-1}=\operatorname{id}_{r(\varphi)}$ and $\varphi^{-1} \circ \varphi=\operatorname{id}_{d(\varphi)}$ where for any set $A \subseteq X, \operatorname{id}_{A}$ denotes the partial Borel isomorphism that is the identity on $A$. The graph of $\varphi$ will be denoted $\Gamma(\varphi)$. We prove in Lemma 2.1 of [MSS] that each Borel set in $C$ can be written as the countable disjoint union of graphs of partial Borel isomorphisms.

For the remainder of this paper we fix a quasi-invariant measure $\mu$ on $X$ and we write $\nu$ for $\nu_{r}$. We normalize $\mu$ to have total mass 1 ; there is no loss in generality in doing this. Recall that a 2-cocycle (with values in $\mathbf{T})$ is a Borel map $s$ from $R^{(2)} \equiv\left\{(z, y, x) \in X^{3} \mid(z, y),(y, x) \in\right.$ $R\}$ such that if $(x, y),(y, z)$, and $(z, t) \in R$, then $s(t, z, x) s(z, y, x)=$ $s(t, y, x) s(t, z, y)$. We fix such a cocycle $s$ and assume that $s$ is skew symmetric in its three variables. (The reader should consult $\S \S 6$ and 7 of [FM1] for the cohomology of equivalence relations.)

A Borel function $a$ on $R$ is called left (resp. right) finite if

$$
\sup _{(x, y) \in R}(|\{z \mid a(x, z) \neq 0\}|+|\{z \mid a(z, y) \neq 0\}|)<\infty
$$

and $a$ is bounded (resp. $D^{-1 / 2} a$ is bounded). Proposition 2.1 of [FM2] shows that if $a$ is a left finite function and if $b$ is a right finite function then the following formulas define bounded operators $L_{a}$ and $R_{b}$ on the Hilbert space $L^{2}(R, \nu)$ :

$$
\begin{aligned}
& L_{a}(\xi)(x, z)=\sum_{y} a(x, y) \xi(y, z) s(x, y, z), \quad \text { and } \\
& R_{b}(\xi)(x, z)=\sum_{y} \xi(x, y) b(y, z) s(x, y, z),
\end{aligned}
$$

where $\xi \in L^{2}(R, \nu)$. Observe that since $a$ is left finite, while $b$ is right finite, the above sums are in fact finite. Using the fact that $s$ is a skew symmetric 2-cocycle, straightforward calculations reveal that $L_{a} L_{b}=L_{a b}, R_{a} R_{b}=R_{b a}, L_{a}^{*}=L_{a^{*}}$, and $R_{a}^{*}=R_{a_{*}}$ where $a b(x, z)=$ $\sum_{y} a(x, y) b(y, z) s(x, y, z), a^{*}(x, y)=\overline{a(y, x)}$, and $a_{*}(x, y)=$ $\overline{a(y, x)} D(y, x)$. Thus, the left finite functions (resp. right finite functions) form a $*$-algebra and $L$ (resp. $R$ ) is a *-representation 
(resp. *-antirepresentation) on $L^{2}(R, \nu)$. The von Neumann algebras generated by the images of $L$ and $R$ will be denoted $M(R, s)$ and $\tilde{M}(R, s)$.

In Proposition 2.5 of [FM2], it is shown that $\tilde{M}(R, s)=M(R, s)^{\prime}$ and that if $\phi_{0}$ denotes the characteristic function of the diagonal $\Delta$ on $R$, then $\phi_{0}$ is a separating and cyclic vector for $M(R, s)$ (and $\tilde{M}(R, s)$ ). Each operator $T$ in $M(R, s)$ (resp. $\tilde{M}(R, s)$ ) has a "matrix" representation. Indeed, if $a(T)$ (resp. $b(T)$ ) denotes $T \phi_{0}$ when $T \in M(R, s)$ (resp. $T \in \tilde{M}(R, s)$ ), then the sum (2.1) converges with $a$ replaced by $a(T)$ (resp. $b$ replaced by $b(T)$ ) and the sum gives $T \xi$. (See Proposition 2.6 of [FM2].)

Let $A(=A(R, s))$ be $\{T \in M(R, s) \mid a(T)$ is supported on $\Delta\}$. Then $A$ is a Cartan subalgebra of $M(R, s)$ in the sense defined in the introduction. The expectation from $M(R, s)$ onto $A$ is given by the formula $E\left(L_{a}\right)=L_{a \cdot \phi_{0}}$ where $a \cdot \phi_{0}$ is the (pointwise) product of $a$ with the characteristic function $\phi_{0}$ of $\Delta$. We will write $E(a)$ for $a \cdot \phi_{0}$. The main result of [FM2], Theorem 1, asserts that if $A$ is a Cartan subalgebra of a von Neumann algebra $M$, then there is a standard equivalence relation $R$, a quasi-invariant measure, and a skew symmetric 2-cocycle $s$, so that $M$ is isomorphic to $M(R, s)$ in such a way that $A$ is carried to $A(R, s)$. Moreover, the measure class of $\mu$ and the cohomology class of $s$ are isomorphism invariants of $M(R, s)$.

Since the characteristic function of the diagonal, $\phi_{0}$, is a cyclic and separating vector for $M(R, s)$, one can compute the ingredients of the Tomita-Takesaki theory for the pair $\left(M(R, s), \phi_{0}\right)$. It turns out that the modular conjugation operator $J$ is given by the formula $(J \xi)(x, y)=\overline{\xi(y, x)} D^{-1 / 2}(y, x)$ and the modular operator $\Delta$ is given by the formula $(\Delta \xi)(x, y)=D(x, y) \xi(x, y)$ (see Proposition 2.8 of [FM2]). According to Proposition 2.9 of [FM2], the algebra $\mathfrak{C}$ generated by $A(R, s)$ and $J A(R, s) J(\subseteq \tilde{M}(R, s))$ turns out to be a maximal abelian subalgebra of the full algebra of operators on $L^{2}(R, \nu)$ and may be identified with $L^{\infty}(R, \nu)$ acting on $L^{2}(R, \nu)$ by pointwise multiplication. This has the very useful consequence that a (closed) subspace of $L^{2}(R, \nu)$ that is invariant under $L_{\tilde{a}}$ and $R_{\tilde{a}}$ for all $a \in L^{\infty}(X, \mu)$, where

$$
\tilde{a}(x, y)=\left\{\begin{array}{cc}
a(x), & x=y \\
0, & x \neq y
\end{array}\right.
$$

must be of the form $L^{2}(Y, \nu)$ for some Borel subset $Y$ of $R$. The Spectral Theorem for Bimodules, Theorem 2.5 of [MSS] is an essential 
generalization of this fact. It asserts that each $\sigma$-weakly closed linear subspace of $M(R, s)$ that is a bimodule over $A(R, s)$, i.e. it is closed under left and right multiplication by elements in $A(R, s)$, must consist of all the operators in $M(R, s)$ whose matrices are supported on some Borel set $B$ in $R$. Conversely, of course, given $B$, the set of operators whose matrices are supported on $B$ is a $\sigma$-weakly closed bimodule. We write $\mathfrak{T}(B)$ for the collection of all such operators. The proof in [MSS] is rather technically involved, but recently Mercer $[\mathbf{M}]$ discovered a very elegant and elementary proof.

If $\varphi$ is a partial Borel isomorphism on $X$ and if $F(\varphi)$ is the characteristic function of its graph $\Gamma(\varphi)$, then $L_{F(\varphi)}$ is a partial isometry in $M(R, s)$ that is zero if and only if $\nu(\Gamma(\varphi))=0$; equivalently, if and only if $\mu(d(\varphi))=0$. The algebraic facts about the partial isometries $L_{F(\varphi)}$ are spelled out in Lemma 2.3 of [MSS]. Basically, in our theory, these partial isometries play the role that matrix units play in algebra. One useful consequence of the Spectral Theorem for Bimodules is that each $\sigma$-weakly closed bimodule is the $\sigma$-weakly closed bimodule generated by the $L_{F(\varphi)}$ contained in it.

The following lemma is proved as Lemma 2.4 of [MSS] and is very useful for showing that certain sets are Borel. We state it here for easy reference as

Lemma 2.1. (1) Let $X_{n}=\{x \| R(x) \mid=n\}, n \leq \infty$. There exist Borel functions

$$
\varphi_{k}: \bigcup\left\{X_{n} \mid n \geq k\right\} \rightarrow \bigcup\left\{X_{n} \mid n \geq k\right\},
$$

$1 \leq k<\infty$, such that for each $x \in X$, the map $k \rightarrow \varphi_{k}(x)$ is a bijection from $\{1,2, \ldots,|R(x)|\}$ (or $\mathbf{N}$, if $|R(x)|=\infty$ ) onto $R(x)$.

(2) For $i=1,2$, let $g_{i}$ be a Borel function defined on Borel subset $Y_{i} \subseteq$ $X$ mapping $Y_{i}$ into $X$. Define $g_{1} \times g_{2}$ by the formula $g_{1} \times g_{2}(x, y)=$ $\left(g_{1}(x), g_{2}(y)\right)$. Then $g_{1} \times g_{2}$ is a Borel map and $\left\{x \in X \mid g_{1}(x)=g_{2}(x)\right\}$ is a Borel subset of $X$.

If $B_{1}$ and $B_{2}$ are subsets of $R$, we write $B_{1} \circ B_{2}=\{(x, y) \mid$ there is a $z \in X$ such that $(x, z) \in B_{1}$ and $\left.(z, y) \in B_{2}\right\}$. Using Lemma 2.1, it is shown in Lemma 3.1 of [MSS] that $B_{1} \circ B_{2}$ is a Borel subset of $R$ if each $B_{i}$ is. In an analogous fashion the $n$-fold composition, $B_{1} \circ B_{2} \circ \cdots \circ B_{n}$, of sets $B_{1}, B_{2}, \ldots, B_{n} \subseteq R$ may be defined. Let $P$ be a 
Borel subset of $R$ and consider the following assertions:

$$
\begin{gathered}
\nu(P \cap \theta(P) \backslash \Delta)=\nu(\Delta \backslash P \cap \theta(P))=0 ; \\
\nu(R \backslash(P \cup \theta(P)))=0 ; \\
\nu(P \circ P \backslash P)=0 ; \\
P \cap \theta(P)=\Delta ; \\
R=P \cup \theta(P) ; \quad \text { and } \\
P \circ P \subseteq P .
\end{gathered}
$$

Assertion $\left(\mathrm{Q}_{1}\right)$ states that $P$ is an antisymmetric relation, and assertion $\left(\mathrm{Q}_{3}\right)$ states that $P$ is a transitive relation. If $P$ satisfies $\left(\mathrm{Q}_{1}\right)$ and $\left(\mathrm{Q}_{3}\right)$, then assertion $\left(\mathrm{Q}_{2}\right)$ asserts that relative to $R, P$ is a total order; i.e., $P$ totally orders each equivalence class determined by $R$. Evidently, assertions $\left(\mathrm{P}_{1}\right),\left(\mathrm{P}_{2}\right)$, and $\left(\mathrm{P}_{3}\right)$ are variants of $\left(\mathrm{Q}_{1}\right),\left(\mathrm{Q}_{2}\right)$, and $\left(\mathrm{Q}_{3}\right)$ modulo null sets. Lemma 3.1 of [MSS] shows that a set satisfying $\left(P_{1}\right),\left(P_{2}\right)$, and $\left(P_{3}\right)$ differs from a set satisfying $\left(Q_{1}\right),\left(Q_{2}\right)$, and $\left(Q_{3}\right)$ by a null set. The point of introducing these conditions is that if $B, B_{1}$, and $B_{2}$ are Borel sets in $R$, then $\mathfrak{T}\left(B_{1}\right) \cdot \mathfrak{T}\left(B_{2}\right) \subseteq \mathfrak{T}(B)$ if and only if $B_{1} \circ B_{2} \subseteq B$ a.e. $\nu ; \mathfrak{T}(B)$ is a subalgebra of $M$ if and only if $B$ satisfies $\left(\mathrm{P}_{3}\right) ; \mathfrak{T}(\theta(B))=\mathfrak{T}(B)^{*} ; \nu\left(R \backslash\left(B_{1} \cup B_{2}\right)\right)=0$ if and only if $\mathfrak{T}\left(B_{1}\right)+\mathfrak{T}\left(B_{2}\right)$ is $\sigma$-weakly dense in $M(R, s)$, and hence $B$ satisfies $\left(\mathrm{P}_{2}\right)$ if and only if $\mathfrak{T}(B)+\mathfrak{T}(B)^{*}$ is $\sigma$-weakly dense in $M(R, s)$; and $B$ satisfies $\left(\mathrm{P}_{1}\right)$ if and only if $\mathfrak{T}(B) \cap \mathfrak{T}(B)^{*}=A(R, s)$. These statements are all proved in Theorem 3.2 of [MSS]. It follows from this that $\mathfrak{T}(B)$ is a von Neumann algebra if and only if $B$ is a sub-equivalence relation of $R$. Moreover, by Theorem 3.4 of [MSS], the map which takes a matrix in $M(R, s)$ to its restriction to $B$ is a faithful, normal, conditional expectation from $M(R, s)$ onto $\mathfrak{T}(B)$. We denote this expectation by $\Phi$. In particular, when $B=\Delta$, then $\Phi=E$.

Let $\mathfrak{A}$ be a $\sigma$-weakly closed subalgebra of a von Neumann algebra $\mathfrak{B}$, suppose that 1 is in $\mathfrak{A}$ and suppose there is a faithful normal expectation $\Psi$ from $\mathfrak{B}$ onto $\mathfrak{A} \cap \mathfrak{A}^{*}$. Then we say that $\mathfrak{A}$ is a subdiagonal subalgebra of $\mathfrak{B}$ (with respect to $\Psi$ ) in the sense of Arveson [A] , in case $\Psi$ is multiplicative on $\mathfrak{A}$, i.e., $\Psi(a b)=\Psi(a) \Psi(b)$ for all $a$ and $b$ in $\mathfrak{A}$, and the $\sigma$-weak closure of $\mathfrak{A}+\mathfrak{A}^{*}$ is $\mathfrak{B}$. Actually, this is not exactly the definition in [A], but it is more serviceable from our perspective. It does no harm to assume that $\mathfrak{A}$ is $\sigma$-weakly closed because the subdiagonal algebras that concern us are maximal in the sense that they are not contained in any larger subalgebra of $\mathfrak{B}$ on which $\Psi$ 
is multiplicative, and any such algebra is $\sigma$-weakly closed. Arveson introduced these algebras in an effort, in part, to overcome some of the difficulties in the Kadison-Singer theory. Our concern with them here arises as follows: Denote by $\mathfrak{P}$ the collection of all Borel subsets $P$ of $R$ that satisfy $\left(\mathrm{P}_{1}\right),\left(\mathrm{P}_{2}\right)$, and $\left(\mathrm{P}_{3}\right)$. Note that we may assume for most purposes that each $P$ in $\mathfrak{P}$ satisfies $\left(\mathrm{Q}_{1}\right),\left(\mathrm{Q}_{2}\right)$, and $\left(\mathrm{Q}_{3}\right)$. Also, write $\mathfrak{P}^{\prime}$ for the collection of all Borel subsets $P$ of $R$ that satisfy $\left(\mathrm{P}_{2}\right),\left(\mathrm{P}_{3}\right)$, and the equation $\nu(\Delta \backslash P)=0$. Then $\mathfrak{T}(P)$ is an algebra for all $P \in \mathfrak{P}^{\prime}$ and $\mathfrak{T}(P \cap \theta(P))=\mathfrak{T}(P) \cap \mathfrak{T}(P)^{*}$ is a von Neumann algebra that is the range of the unique faithful normal expectation $\Phi$ on $M(R, s)$ as noted above. Theorem 3.5 of [MSS] asserts that the map $P \rightarrow \mathfrak{T}(P)$ is a bijection between $\mathfrak{P}^{\prime}$ (where we identify two sets that differ by a $\nu$-null set) and the set of all maximal subdiagonal subalgebras of $M$ that contain $A(R, s)$. Moreover (and this is Corollary 3.6 of [MSS]), each $\mathfrak{T}(P), P \in \mathfrak{P}$, is triangular and each $\sigma$-weakly closed maximal triangular subalgebra of $M(R, s)$ with diagonal $A(R, s)$ is of the form $\mathfrak{T}(P), P \in \mathfrak{P}$. In particular, each $\sigma$-weakly closed maximal triangular subalgebra of $M(R, s)$ with diagonal $A(R, s)$ is a maximal subdiagonal subalgebra of $M(R, s)$ with respect to the faithful normal expectation $E$ from $M(R, s)$ onto $A(R, s)$.

3. Nest subalgebras, analytic subalgebras, and partial orders. In this section we provide criteria for deciding when one of the algebras $\mathfrak{T}(P)$ is an analytic subalgebra or a nest subalgebra of $M(R, s)$. Henceforth, we will write simply $M$ for $M(R, s)$ and $A$ for $A(R, s)$.

We begin by recalling some basic facts from [LM] where the theory of analytic subalgebras of von Neumann algebras was first introduced and studied systematically.

Suppose that $\mathfrak{R}$ is a von Neumann algebra and that $\alpha=\left\{\alpha_{t}\right\}_{t \in \mathbf{R}}$ is a $\sigma$-weakly continuous representation of the real line $\mathbf{R}$ as a group of $*$-automorphisms of $\mathfrak{R}$. We will call such an $\alpha$ a flow on $\mathfrak{R}$. For $x \in \mathfrak{R}$, we let $\mathrm{sp}_{\alpha}(x)$ denote the Arveson spectrum of $x$ with respect to $\alpha$ (see [A2]). The analytic subalgebra of $\mathfrak{R}$ with respect to $\alpha, H^{\infty}(\alpha)$, defined in the introduction coincides with $\left\{x \in \mathfrak{R} \mid \operatorname{sp}_{\alpha}(x) \subseteq[0, \infty)\right\}$. (It requires a little proof to see that in fact $H^{\infty}(\alpha)$ is an algebra.) In Theorem 3.15 of [LM] it is shown that whenever there exists a faithful normal expectation $\Phi$ from $\mathfrak{R}$ onto $\mathfrak{R}^{\alpha}:=\left\{x \in \mathfrak{R} \mid \operatorname{sp}_{\alpha}(x) \subseteq\{0\}\right\}$, the fixed point algebra of $\alpha$, such that $\Phi \circ \alpha_{t}=\Phi$ for all $t \in \mathbf{R}$, then $H^{\infty}(\alpha)$ is a maximal subdiagonal algebra in $\mathfrak{R}$ with respect to $\Phi$. When $\alpha$ is an inner flow, i.e., when $\alpha_{t}=\operatorname{Ad} u_{t}$ for a strongly continuous unitary group $\left\{u_{t}\right\}_{t \in \mathbf{R}}$ in $\mathfrak{R}$, then $H^{\infty}(\alpha)$ is a nest subalgebra of $\mathfrak{R}$ in the sense 
that there is a nest $\mathfrak{N}$ of projections in $\mathfrak{R}$ such that $H^{\infty}(\alpha)=\{x \in$ $\mathfrak{R} \mid(1-p) x p=0$, for all $p \in \mathfrak{R}\}$. In fact, given $\alpha_{t}=\operatorname{Ad} u_{t}$ with $u_{t}=\int_{-\infty}^{\infty} e^{i \lambda t} d E(\lambda)$, one may take $\mathfrak{N}=\{E([t, \infty)) \mid t \in \mathbf{R}\}$. Conversely, if $H^{\infty}(\alpha)$ is a nest subalgebra, then $\alpha$ is inner. Indeed, the projections in the nest can be used to build a spectral measure on $\mathbf{R}$ whose FourierStieltjes transform implements $\alpha$. For the details of this, the reader should consult $\S 4.2$ of [LM].

We continue with the notation of $\S 2$; we let $\mathfrak{P}(a)$ denote the collection of $P \in \mathfrak{P}$ such that $\mathfrak{T}(P)$ is an analytic subalgebra of $M$ (with respect to some flow) and we let $\mathfrak{P}(n)$ denote the collection of $P \in \mathfrak{P}$ such that $\mathfrak{T}(P)$ is a nest subalgebra of $M$. By the preceding discussion, we have $\mathfrak{P}(n) \subseteq \mathfrak{P}(a) \subseteq \mathfrak{P}$. As we shall see presently, each $P \in \mathfrak{P}(a)$ is determined by a 1-cocycle for $R$ with values in $\mathbf{R}$. It will turn out that such a $P$ belongs to $\mathfrak{P}(n)$ if and only if the cocycle is a coboundary. In addition, we shall see in $\S 4$ that $\mathfrak{P}(a)$ may be properly contained in $\mathfrak{P}$. Nevertheless, as we shall see presently, for every $P \in \mathfrak{P}, \mathfrak{T}(P)$ is contained in a direct integral of nest algebras.

We will require some of the cohomology theory of [FM1]. Let $G$ be a separable locally compact abelian group with operation written multiplicatively. (When $G=\mathbf{Z}$ or $\mathbf{R}$, we will write the operation additively.) We denote by $Z^{1}(R, G)$ the collection of all measurable functions $c$ on $R$ with values in $G$ such that there is a conull Borel set $X_{0} \subseteq X$ (depending on $c)$ so that for all $(x, y),(y, z) \in R \cap\left(X_{0} \times X_{0}\right)$ we have $c(x, z)=c(x, y) c(y, z)$. Such a function is called a cocycle. If there is a function $b: X \rightarrow G$ such that $c(x, y)=b(x) b(y)^{-1}$ a.e. $(\nu)$, then $c$ is called a coboundary and we write $c \in B^{1}(R, G)$. If one views $R$ as a groupoid, then a cocycle simply is a homomorphism from $R$ into $G$ and so, under pointwise multiplication, the cocycles form a group with the coboundaries forming a subgroup. Theorem 2 of [FM2] implies that $Z^{1}(R, \mathbf{T})$ is isomorphic to the subgroup of $\operatorname{Aut}(M)$ consisting of those automorphisms that fix $A$ elementwise. For $c \in Z^{1}(R, T)$, the corresponding automorphism $\gamma$ is implemented by the unitary operator $U$ given by the equation $(U \xi)(x, y)=c(x, y) \xi(x, y), \xi \in$ $L^{2}(R, \nu)$. It follows that if $a$ is a left finite function on $R$, then $\gamma\left(L_{a}\right)=$ $U L_{a} U^{-1}=L_{c \cdot a}$ where $(c \cdot a)(x, y)=c(x, y) a(x, y)$. Moreover, there is a bijective correspondence between the flows $\alpha$ on $M$ with the property that $A \subseteq M^{\alpha}$, where $M^{\alpha}=\left\{T \in M \mid \alpha_{t}(T)=T\right.$ for all $\left.t \in \mathbf{R}\right\}$ and $Z^{1}(R, \mathbf{R})$. The flow $\alpha$ that corresponds to $d \in Z^{1}(R, \mathbf{R})$ is implemented by $\left\{U_{t}\right\}_{t \in \mathbf{R}}$ where $\left(U_{t} \xi\right)(x, y)=\exp (i t d(x, y)) \xi(x, y), \xi \in$ $L^{2}(R, \nu)$. So, for a left finite function $a$ on $R, \alpha_{t}\left(L_{a}\right)=L_{a_{t}}$ where $a_{t}(x, y)=\exp (i t d(x, y)) a(x, y)$. (See Theorem 5 of [FM2].) 
For $d \in Z^{1}(R, \mathbf{R})$ we write $P(d)=\{(x, y) \in R \mid d(x, y) \geq 0\}$. If one views $d \in Z^{1}(R, \mathbf{R})$ as an $\mathbf{R}$-valued homomorphism on the groupoid $R$, then since $P(d)$ is the inverse image under $d$ of the semigroup $\{t \mid t \geq 0\}$, one should expect that $P(d)$ is a subsemigroupoid of $R$, i.e., a partial order. The following proposition affirms this, modulo null sets of course. It is proved as Lemma 3.7 and Proposition 3.8 of [MSS].

Proposition 3.1. For $d \in Z^{1}(R, \mathbf{R}), P(d)$ satisfies $\left(\mathrm{P}_{2}\right),\left(\mathrm{P}_{3}\right)$, and the equation $\nu(\Delta \backslash P(d))=0$. Thus $P(d) \in \mathfrak{P}^{\prime}$. Conversely, suppose $P \in \mathfrak{P}^{\prime}$, suppose $d \in Z^{1}(R, \mathbf{R})$, and let $\alpha$ be the corresponding flow. Then $\mathfrak{T}(P)=H^{\infty}(\alpha)$ if and only if $P=P(d)$ a.e. $\nu$.

Corollary 3.2. A set $P$ in $\mathfrak{P}$ lies in $\mathfrak{P}(a)$ if and only if there is a $d$ in $Z^{1}(R, \mathbf{R})$ with $\Delta=d^{-1}(0)$ such that $P=P(d)$.

The following lemma is known, but we include a proof for completeness.

LEMMA 3.3. Let $\alpha$ be a flow on $M$ corresponding to a cocycle $d \in$ $Z^{1}(R, \mathbf{R})$. Then $\alpha$ is inner if and only if $d \in B^{1}(R, \mathbf{R})$; i.e., if and only if there is a Borel function $g: X \rightarrow \mathbf{R}$ such that $d(x, y)=g(y)-g(x)$ a.e. $\nu$.

Proof. Suppose $g$ is a real-valued Borel function on $X$ and let $V_{t}=$ $L_{\exp (-i t g)}, t \in \mathbf{R}$. Then each $V_{t}$ lies in $M$ and for $L_{a} \in M$ and $\xi \in$ $L^{2}(R, \nu)$ we see that

$$
\begin{aligned}
\left(V_{t} L_{a} V_{t}^{*} \xi\right)(x, y) & =e^{-i \operatorname{tg}(x)} \sum_{z} a(x, z) e^{i \operatorname{tg}(z)} \xi(z, y) s(x, z, y) \\
& =\left(L_{a_{t}} \xi\right)(x, y),
\end{aligned}
$$

where $a_{t}(x, y)=e^{i t(g(y)-g(x))} a(x, y)$. Hence, if $d \in B^{1}(R, \mathbf{R}), \alpha$ is inner implemented by $\left\{V_{t}\right\}_{t \in \mathbf{R}}$. On the other hand, if $\alpha$ is an inner flow associated with $d \in Z^{1}(R, \mathbf{R})$, then since $A \subseteq M^{\alpha}$ and $A^{\prime} \cap M=$ $A, \alpha$ is implemented by a unitary group $\left\{L_{\exp (-i t g)}\right\}_{t \in \mathbf{R}}$ where $g$ is a real-valued Borel function on $X$. The above calculation shows that $d(x, y)=g(y)-g(x)$ a.e.

Corollary 3.4. A set $P \in \mathfrak{P}$ belongs to $\mathfrak{P}(n)$ if and only if there is a real-valued Borel function $g$ on $X$ such that $P=\{(x, y) \in R \mid g(y) \geq$ $g(x)\}$ a.e. $\nu$. In this event, $g$ is essentially one-to-one; i.e., $\nu(\{(x, y) \in$ $R \mid g(x)=g(y), x \neq y\})=0$. 
Evidently, $\mathfrak{T}(P)$ is always contained in a nest algebra in a trivial fashion. Indeed, $\mathfrak{T}(P)=M \cap \mathfrak{A}$, where $\mathfrak{A}$ is the nest algebra on $L^{2}(R, \nu)$ associated with the nest whose elements are zero, the identity, and the projection onto $L^{2}(P, \nu)$, and this representation is, in an obvious sense, trivial. We want a representation that carries more information. To this end, observe that since $J A J$ is a masa in $M^{\prime}$, the von Neumann algebra $[M, J A J]$ generated by $M$ and $J A J$ coincides with $(J A J)^{\prime}$, a type I von Neumann algebra of infinite multiplicity. We will show that the weakly closed algebra on $L^{2}(R, \nu)$ generated by $\mathfrak{T}(P)$ and $J A J$, which we denote by $[\mathfrak{T}(P), J A J]$, is a nest subalgebra of $(J A J)^{\prime}$ and $\mathfrak{T}(P)=M \cap[\mathfrak{T}(P), J A J]$. We express our results in terms of automorphism groups, exploiting the relation between automorphism groups and nest algebras outlined at the beginning of this section.

We begin by noting that if $d$ is an arbitrary real-valued Borel function on $R$, then $d$ determines a unitary representation of $\mathbf{R},\left\{U_{t}^{d}\right\}_{t \in \mathbf{R}}$, on $L^{2}(R, \nu)$ by the formula $\left(U_{t}^{d} \xi\right)(x, y)=\exp (i t d(x, y)) \xi(x, y), \xi \in$ $L^{2}(R, \nu)$. Evidently, $\left\{U_{t}^{d}\right\}_{t \in \mathbf{R}}$ lies in the masa $\mathfrak{C}$ generated by $A$ and $J A J$; and conversely, every one parameter unitary group that lies in $\mathfrak{C}$ is of the form $\left\{U_{t}^{d}\right\}_{t \in \mathbf{R}}$ for a suitable function $d$. (Recall that $\mathfrak{C}$ may be identified with $L^{\infty}(R, \nu)$ acting on $L^{2}(R, \nu)$ via pointwise multiplication.) Given $d$, set $\alpha_{t}^{d}=\operatorname{Ad}\left(U_{t}^{d}\right)$ acting on all of $\mathscr{L}\left(L^{2}(R, \nu)\right)$. Then as was noted at the beginning of this section, $H^{\infty}\left(\alpha^{d}\right)$ is the nest algebra determined by the spectral projections $\left\{E^{d}[s, \infty)\right\}_{s \in \mathbf{R}}$ for $\left\{U_{t}^{d}\right\}_{t \in \mathbf{R}}$. These, in turn, lie in $\mathfrak{C}$ and therefore are given by multiplication operators; viz., $E^{d}[s, \infty)$ is multiplication by the characteristic function of $\{(x, y) \mid d(x, y) \geq s\}$. On the other hand, a nest algebra determined by a nest in $\mathfrak{C}$ must be of the form $H^{\infty}\left(\alpha^{d}\right)$ for some function $d$. It should be noted that different $d$ 's may give rise to the same nest algebra. Also, we note that given $d, M \cap H^{\infty}\left(\alpha^{d}\right)=\mathfrak{T}(P)$ where

$$
P=\{(x, y) \in R \mid d(x, z) \geq d(y, z) \text { for all } z \sim x \sim y\} .
$$

This is easy to see by writing out the matrix for $\alpha_{t}^{d}(T), T \in M$, and noting that the resulting matrix is pointwise analytic in $t$, i.e., $a\left(\alpha_{t}^{d}(T)\right)(x, y)$ lies in $H^{\infty}(\alpha)$ as a function of $t$ for each $(x, y) \in R$, if and only if $a(T)$ is supported on $P$. Note that if $d$ is a cocycle, then $P=\{(x, y) \in R \mid d(x, y) \geq 0\}$. In general, given $d, P$, defined by equation (3.1), is a partial order, but it seems difficult to say much about its properties; in particular, we are unable to decide in terms of $d$ when $P$ satisfies $\left(\mathrm{P}_{2}\right)$. 
THEOREM 3.5. For $P \in \mathfrak{P}^{\prime}$, there is a real-valued Borel function $d$ on $R$ such that $[\mathfrak{T}(P), J A J]$ is the nest subalgebra of $(J A J)^{\prime},(J A J)^{\prime} \cap$ $H^{\infty}\left(\alpha^{d}\right)$, and $\mathfrak{T}(P)=M \cap H^{\infty}\left(\alpha^{d}\right)$.

Proof. We may assume that $\Delta \subseteq P$ and that $P$ satisfies $\left(\mathrm{Q}_{2}\right)$ and $\left(\mathrm{Q}_{3}\right)$. We write $L^{2}(R, \nu)$ as a direct integral as in $\S 2$ of [MSS], but here we disintegrate $\nu$ via $\pi_{r}$. We regard the direct integral

$$
\int^{\oplus} l^{2}\left(\pi_{r}^{-1}(y)\right) d \mu(y)
$$

as consisting of fields $\left\{\xi_{y}\right\}_{y \in X}$ of functions given by the formula

$$
\xi_{y}(x, z)= \begin{cases}\xi(x, y), & z=y \\ 0, & z \neq y\end{cases}
$$

where $\xi \in L^{2}(R, \nu)$ and we define $W$ from $L^{2}(R, \nu)$ to the

$$
\int^{\oplus} l^{2}\left(\pi_{r}^{-1}(y)\right) d \mu(y)
$$

by the formula

$$
(W \xi)_{y}(x, z)= \begin{cases}\xi(x, y), & y=z, \\ 0, & y \neq z,\end{cases}
$$

This $W$ is a Hilbert space isomorphism with inverse given by the equation $\left(W^{-1} \xi\right)(x, y)=\xi_{y}(x, y), \xi=\left\{\xi_{y}\right\}_{y \in X} \in \int^{\oplus} l^{2}\left(\pi_{r}^{-1}(y)\right) d \mu(y)$. It is easy to see that $W(J A J) W^{-1}$ is the algebra of diagonal operators $\int^{\oplus} l^{2}\left(\pi_{r}^{-1}(y)\right) d \mu(y)$, so that every $T \in M$ is decomposable. In fact, for $T \in M$ we have

$$
\left(W T W^{-1}\right)_{y} \xi_{y}(x, z)= \begin{cases}\sum_{u} a(T)(x, u) s(x, u, y) \xi(u, y), & y=z, \\ 0, & y \neq z,\end{cases}
$$

For $x \in X$, we write $(-\infty, x]=\{y \mid(y, x) \in P\}$ by definition. For $y \in X$ and $x \in R(y)$, set

$$
\mathfrak{H}(x ; y)=\left\{\xi_{y} \in l^{2}\left(\pi_{r}^{-1}(y)\right) \mid \operatorname{supp} \xi_{y} \subseteq(-\infty, x]\right\}
$$

and let $\mathscr{L}(y)$ be the complete nest generated by $\{\mathfrak{H}(x ; y)\}_{x \in R(y)}$. (Note that for each $y, \mathfrak{H}\left(x_{1} ; y\right) \subseteq \mathfrak{H}\left(x_{2} ; y\right)$ if and only if $\left(x_{1}, x_{2}\right) \in P$, so by $\left(\mathrm{Q}_{2}\right),\{\mathfrak{H}(x ; y)\}_{x \in R(y)}$ is a nest. Note, too, that $\bigcap_{x \in R(y)} \mathfrak{H}(x ; y)=\{0\}$ while $\bigcup_{x \in R(y)} \mathfrak{H}(x, y)$ is dense in $l^{2}\left(\pi_{r}^{-1}(y)\right)$. Thus $\{0\}$ and $l^{2}\left(\pi^{-1}(y)\right)$ 
are contained in $\mathscr{L}(y)$.) Choose a sequence $\left\{T_{n}\right\}_{n=1}^{\infty}$ that is $\sigma$-weakly dense in $\mathfrak{T}(P)$ such that the matrices $a\left(T_{n}\right)$ separate the points of $P$. For each $y \in X$, set $\mathfrak{T}_{y}$ equal to the weakly closed algebra generated by $\left\{\left(W T_{n} W^{-1}\right)_{y}\right\}_{n=1}^{\infty}$. Then it is easy to see that $\mathfrak{T}_{y}=\operatorname{alg}(\mathscr{L}(y))=$ alg lat $\left(\mathfrak{T}_{y}\right)$ for all $y$. The key ingredient of the proof is this assertion: $T \in M$ lies in $\mathfrak{T}(P)$ if and only if $\left(W T W^{-1}\right)_{y} \in \operatorname{Alg}(\mathscr{L}(y))$ a.e. Indeed, if $T$ belongs to $\mathfrak{T}(P)$, if $y \in X$, and if $\xi_{y} \in \mathfrak{H}(x ; y)$, then

$$
\left(W T W^{-1}\right)_{y} \xi_{y}(u, y)=\sum_{v} a(T)(u, v) s(u, v, y) \xi(v, y) .
$$

If $(x, u) \in P \backslash(P \cap \theta(P)), u \in R(y)$, then since the sum runs over those $v$ such that $(u, v) \in P$, and since $\xi(v, y)=0$ for each such $v$, the sum vanishes; i.e., $\left(W T W^{-1}\right)_{y} \xi_{y} \in \mathfrak{H}(x ; y)$. Thus if $\left(W T W^{-1}\right)_{y} \in$ $\operatorname{Alg}(\mathscr{L}(y))$ a.e. $\mu$, then for each fixed $y$ outside of an exceptional null set, the operator on $l^{2}\left(\pi_{r}^{-1}(y)\right)$ represented by the matrix $a(T)$ leaves each $\mathfrak{H}(x ; y)$ invariant. This means that for $x, z \in R(y), a(T)(x, z)=$ 0 when $(z, x) \in P \backslash(P \cap \theta(P))$. In other words, except for a null set of $y$,

$$
\operatorname{supp} a(T) \cap(R(y) \times R(y)) \subseteq P \cap(R(y) \times R(y)),
$$

and so $T \in \mathfrak{T}(P)$, completing the proof of the assertion.

Thus, in the notation and terminology of [AFG] and [GL], $W \mathfrak{T}(P) W^{-1} \sim \int^{\oplus} \mathfrak{T}_{y} d \mu(y)$ and if $\mathscr{L}$ is the lattice of the algebra generated by $\mathfrak{T}(P)$ and $J A J$, then $W \mathscr{L} W^{-1}=\int^{\oplus} \mathscr{L}(y) d \mu(y)$. Since each $\mathfrak{T}_{y}$ is reflexive, Proposition 5.6 of [AFG] implies that $[\mathfrak{T}(P), J A J]$ is reflexive. So, since $[\mathfrak{T}(P), J A J]$ contains the masa $\mathfrak{C}$ in $\mathscr{L}\left(L^{2}(R, \nu)\right)$ generated by $A$ and $J A J$, we conclude from Theorem 4.1 of [GL] and from the fact that $W[\mathfrak{T}(P), J A J] W^{-1}$ is the direct integral of nest algebras that $[\mathfrak{T}(P), J A J]$ is a nest subalgebra of $J A J^{\prime}$. The projections in this nest all commute with $\mathfrak{C}$, and therefore they lie in $\mathfrak{C}$. By the discussion preceding the statement of this theorem, there is a real-valued function $d$ on $R$ such that $[\mathfrak{T}(P), J A J]=(J A J)^{\prime} \cap H^{\infty}\left(\alpha^{d}\right)$. Since $\mathfrak{T}(P)=M \cap[\mathfrak{T}(P), J A J]$, as we just showed, and since $M \subseteq(J A J)^{\prime}$, it follows that $\mathfrak{T}(P)=M \cap H^{\infty}\left(\alpha^{d}\right)$.

When $M=\mathscr{L}(\mathscr{H})$, a Cartan subalgebra $A$ is the subalgebra of operators with diagonal matrices with respect to some orthonormal basis. In this case, Theorem 3.2.1 in [KS] asserts that given a maximal triangular algebra $\mathfrak{T}$ with diagonal $A$, there is a total ordering on the basis such that $\mathfrak{T}$ consists of all the operators in $M$ whose matrices with respect to the basis are upper triangular; i.e., $\mathfrak{T}$ is a special kind of nest algebra in $M$. The following theorem should be viewed as a direct generalization of this result. 
Theorem 3.6. Suppose that $M$ is type I. Then $\mathfrak{P}=\mathfrak{P}(a)=\mathfrak{P}(n)$. Hence when $M$ is type I, every maximal subdiagonal algebra in $M$, with respect to $E$, is a nest subalgebra of $M$.

Proof. Fix $P \in \mathfrak{P}$; we need only show $P \in \mathfrak{P}(n)$. For this, it suffices, by Corollary 3.4, to produce a Borel function $g: X \rightarrow \mathbf{R}$ such that $P=\{(x, y) \in R \mid g(y) \geq g(x)\}$. Since $R$ is type $\mathrm{I}$, its fundamental class is trivial by Proposition 7.3 of [FM1]. Consequently, the functions $\varphi_{k}$ in Lemma 2.1(1) can be chosen so that $\varphi_{k}(x)=\varphi_{k}(y)$ for almost all $(x, y) \in R$. We proceed to define maps $f_{k}: \cup_{n>k} X_{n} \rightarrow \mathbf{Q}$ with the property that $\left(\varphi_{k}(x), \varphi_{j}(x)\right) \in P$ if and only if $f_{k}(x) \leq f_{j}(x)$, and $f_{k}(x)=f_{j}(x)$ only when $j=k$. (Recall $X_{n}=\{x \| R(x) \mid=n\}$.) The following definition is based on Theorem 2.5 of [R]. First enumerate $Q \backslash \mathbf{Z}$ as $\left\{r_{i}\right\}_{i=1}^{\infty}$. Set $f_{1} \equiv 0$, and suppose that $f_{1}, f_{2}, \ldots, f_{n-1}$ have been defined. For each $x \in X$ one and only one of the following assertions holds:

(1) $\left(\varphi_{j}(x), \varphi_{n}(x)\right) \in P$ for all $j<n$;

(2) $\left(\varphi_{n}(x), \varphi_{j}(x)\right) \in P$ for all $j<n$; and

(3) There is a $j<n$ such that $\left(\varphi_{j}(x), \varphi_{n}(x)\right) \in P$ and there is an $i<n$ such that $\left(\varphi_{n}(x), \varphi_{i}(x)\right) \in P$.

If (1) holds, set $f_{n}(x)=n$, while if (2) holds, set $f_{n}(x)=-n$. If (3) holds, define $f_{n}(x)$ to be the first $r_{i}$ that is in the same relation to $f_{1}(x), \ldots, f_{n-1}(x)$ as $\varphi_{n}(x)$ is to $\varphi_{1}(x), \ldots, \varphi_{n-1}(x)$, where the order among the $\varphi_{j}(x)$ 's is the order induced by $P$. It is easy to see that the $f_{n}$ satisfy the desired properties.

For each $x \in X$, let $n(x)$ be the integer $n$ such that $\varphi_{n}(x)=x$ and set $g(x)=f_{n(x)}(x)$. Let $B_{n}=\left\{(x, y) \in R \mid \varphi_{n}(x)=\varphi_{n}(y)\right\}, n \geq 1$, and observe that $B_{n}$ is Borel and conull. By definition of the $f_{n}$, it is evident that for $(x, y) \in B:=\bigcap B_{n}, f_{n}(x)=f_{n}(y)$ for all $n \geq 1$. So for $(x, y) \in P \cap B, \varphi_{n(x)}(y)=\varphi_{n(x)}(x)=x$, and $\varphi_{n(y)}(y)=y$. Hence $\left(\varphi_{n(x)}(y), \varphi_{n(y)}(y)\right)$ lies in $P$. This implies that $g(x)=f_{n(x)}(x)=$ $f_{n(x)}(y) \leq f_{n(y)}(y)=g(y)$. Therefore, $g(y) \geq g(x)$ for $\nu$-almost all $(x, y) \in P$. Equality holds, on $P \cap B$, if and only if $n(x)=n(y)$, and this can occur only if $x=y$.

All that remains to complete the proof is to show that $g$ is a Borel map into $\mathbf{Q}$. Since $g=f \circ \tilde{n}$ where $f: \mathbf{Z}_{+} \times X \rightarrow \mathbf{Q}$ is given by the formula $f(m, x)=f_{m}(x)$ and $\tilde{n}: X \rightarrow \mathbf{Z}_{+} \times X$ is given by the formula $\tilde{n}(x)=(n(x), x)$, it suffices to prove that $f$ and $n$ are Borel. But for $m \geq 0,\{x \in X \mid n(x)=m\}=\left\{x \in X \mid \varphi_{m}(x)=x\right\}$. Thus $n$ is Borel. To 
prove that $f$ is Borel, fix $r \in \mathbf{Q}$ and note that

$$
f^{-1}(r)=\left\{(m, x) \mid f_{m}(x)=r\right\}=\bigcup_{m}\left\{(m, x) \mid f_{m}(x)=r\right\} .
$$

So, it suffices to show that the set $c(m, r):=\left\{x \in X \mid f_{m}(x)=r\right\}$ is a Borel set for all $m \geq 1, r \in \mathbf{Q}$. From the definition of $f_{m}$, one sees that $c(m, r)$ is the intersection of finitely many sets of the form $\left\{x \in X \mid\left(\varphi_{i}(x), \varphi_{j}(x)\right) \in P\right\}$. Since each of these sets is Borel by Lemma 2.4(2), it follows that $c(m, r)$ is Borel. This completes the proof.

Theorem 6 of [FM1] asserts that if $d \in Z^{1}(R, \mathbf{R})$ is essentially bounded, then $d$ is a coboundary. The following is a strengthened version of this fact. It follows from general principles of the cohomology theory of groupoids. However, we give an elementary, self-contained proof here.

Proposition 3.7. Suppose $d \in Z^{1}(R, \mathbf{R})$ and that there is a conull set $B \subseteq X$ with the properties that $R(B)$ is conull and that the restriction of $d$ to $R \cap(B \times B)$ is bounded. Then $d$ is a coboundary.

Proof. Fix a countable group $G$ acting on $X$, so that $R=R_{G}$ (see [FM1, Theorem 1]), and write $g \cdot x$ for the translate of $x \in X$ by $g \in G$. Then $R=R_{G}=\{(x, g \cdot x) \mid g \in G, x \in X\}$. For $g \in G$ and $n \in \mathbf{Z}$, set $c(g, n)=\{(x, g \cdot x)|| d(x, g \cdot x) \mid \leq n\}$. Evidently, $c(g, n)$ is the graph of a partial Borel isomorphism. Let $\mathscr{N}$ be the set of all partial Borel isomorphisms obtained in this manner. Then $\mathscr{N}$ is countable and $\bigcup\{\varphi(B) \mid \varphi \in \mathscr{N}\}=R(B)$-a conull set. Also, we have $\varphi^{-1} \in \mathscr{N}$ for all $\varphi \in \mathscr{N}$, and for each $\varphi \in \mathscr{N}, \sup \{d(x, y) \mid(x, y) \in \Gamma(\varphi)\}$ is finite. Denote this supremum by $r(\varphi)$. Writing $B_{0}=B$, let $\left\{B_{j}\right\}$ be a maximal family of pairwise disjoint Borel subsets of $X$, each having positive measure, such that for each $j$, there is a $\psi_{j} \in \mathscr{N}$ satisfying $\psi_{j}^{-1}\left(B_{j}\right) \subseteq$ $B$. (Such a family exists by Zorn's Lemma.) Since $\bigcup\{\varphi(B) \mid \varphi \in \mathscr{N}\}$ is conull, so is $\bigcup_{j=0}^{\infty} B_{j}$. Set $C_{n}=\bigcup_{j=0}^{n} B_{j}, n \geq 0$, and set $C_{\infty}=\bigcup_{j=0}^{\infty} B_{j}$.

We show that $d \mid R \cap\left(C_{n} \times C_{n}\right)$ is essentially bounded for each $n$. By Theorem 6 of [FM1], then, each of these restrictions is a coboundary. We show, too, that the bounding functions for each of these coboundaries may be chosen in a consistent way yielding a global function that shows that $d$ is a coboundary. Let $M$ be a bound for $d \mid R \cap(B \times B)$, fix $n$, choose $x, y \in C_{n}$, and choose $i, j \leq n$ and $z, w \in B$ with $x=\psi_{i}(z)$ 
and $y=\psi_{j}(w)$. Then

$$
\begin{aligned}
|d(x, y)| & =\left|d\left(\psi_{i}(z), \psi_{j}(w)\right)\right| \\
& \leq\left|d\left(\psi_{i}(z), z\right)\right|+|d(z, w)|+\left|d\left(w, \psi_{j}(w)\right)\right| \\
& \leq r\left(\psi_{i}^{-1}\right)+M+r\left(\psi_{j}\right)<\infty .
\end{aligned}
$$

This shows that $d$ is bounded on $R \cap\left(C_{n} \times C_{n}\right)$. By Theorem 6 of [FM1], applied to $R \cap\left(C_{n} \times C_{n}\right)$, we may find functions $g_{n}^{\prime}: C_{n} \rightarrow \mathbf{R}$ such that

$$
d(x, y)=g_{n}^{\prime}(y)-g_{n}^{\prime}(x), \quad(x, y) \in R \cap\left(C_{n} \times C_{n}\right) .
$$

Observe that for $x, y \in B_{0} \subseteq C_{n}, n \geq 0, g_{n}^{\prime}(y)-g_{n}^{\prime}(x)=d(x, y)=$ $g_{0}^{\prime}(y)-g_{0}^{\prime}(x)$. Hence, if we set $f_{n}=g_{0}^{\prime}(y)-g_{0}^{\prime}(x)$, we see that $f_{n}$ is invariant; i.e., $f_{n}(x)=f_{n}(y)$ for all $(x, y) \in R \cap\left(B_{0} \times B_{0}\right)$. Next, we define functions $f_{n}^{\prime}, n \geq 0$, on $C_{n}$ by setting $f_{n}^{\prime}(x)=f_{n}(y)$ for any $y \in R(x) \cap B_{0}$. Since $C_{n} \subseteq R\left(B_{0}\right)$ and since the $f_{n}$ are invariant, it follows that the $f_{n}^{\prime}$ are well defined and invariant. It is easy to check that they are Borel functions. On $C_{n}, n \geq 1$, define $g_{n}$ to be $g_{n}^{\prime}-f_{n}^{\prime}$. Then by the invariance of $f_{n}^{\prime}, g_{n}(y)-g_{n}(x)=g_{n}^{\prime}(y)-g_{n}^{\prime}(x)=d(x, y)$. Now fix $m>n$, and let $h_{m, n}$ be defined on $C_{n}$ by the formula

$$
h_{m, n}=g_{m} \mid C_{n}-g_{n}
$$

The argument just presented shows that $h_{m, n}$ is invariant and that $h_{m, n}$ vanishes on $B_{0}$. Since the saturation of $B_{0}$ is almost all of $X$, it follows that $h_{m, n}=0$ a.e.; i.e., $g_{m} \mid C_{n}=g_{n}$ a.e. Thus, there is an almost everywhere, well-defined function $g$ on $X$ such that $g$ agrees with $g_{n}$ on $C_{n}$ and $g(y)-g(x)=d(x, y)$ a.e. This shows that $d \in B^{1}(R, \mathbf{R})$ as required.

Let $P \in \mathfrak{P}^{\prime}$; i.e., assume that $P$ satisfies $\left(\mathrm{P}_{2}\right)$ and $\left(\mathrm{P}_{3}\right)$ of $\S 3$ and the equation $\nu(\Delta \backslash P)=0$. If $F \subseteq X$ is a Borel set, we write $P(F)=$ $\{x \in X \mid$ there is $y \in F$ such that $(y, x) \in P\}$, and we say that $F$ is $P$-increasing, or increasing relative to $P$, or simply increasing if $P$ is understood, in case $\mu(P(F) \backslash F)=0$. For an example of an increasing set, let $P=P(d)$, where $d \in B^{1}(R, \mathbf{R})$, choose $g$ so that $d=\delta g$ and let $r \in \mathbf{R}$. Then the set $\{x \in X \mid g(x) \geq r\}$ is increasing. The following lemma shows a partial converse. In a sense it is an analogue of the decomposition of a Markov process into recurrent and transient parts.

LemMA 3.8. Suppose that $d \in Z^{1}(R, \mathbf{R})$, that $P=P(d)$, and that $F \subseteq X$ is increasing. Let $F_{\infty}=\{x \in X \mid R(x) \cap F$ is empty $\}$, let 
$F_{-\infty}=\{x \in X \mid R(x) \subseteq F\}$, and let $F_{0}=X \backslash\left(F_{\infty} \cup F_{-\infty}\right)$. Then $F_{\infty}, F_{-\infty}$, and $F_{0}$ are all invariant Borel sets and the function

$$
g(x):=\sup \{d(y, x) \mid y \in F,(x, y) \in R\}
$$

is an almost everywhere defined (with respect to $\mu$ ) function on $F_{0}$ satisfying

$$
d(x, y)=g(y)-g(x), \quad(x, y) \in R \cap\left(F_{0} \times F_{0}\right) .
$$

In particular, if $\nu(X \backslash R(F))=\nu(X \backslash R(X \backslash F))=0$, then $d \in B^{1}(R, \mathbf{R})$.

Proof. Evidently, the sets $F_{\infty}, F_{-\infty}$, and $F_{0}$ are invariant. The fact that they are Borel is most easily seen if one writes $R=R_{G}$ for a suitable countable group of transformations. Then $F_{\infty}$ is $\bigcap_{t \in G} t \cdot(X \backslash F)$ while $F_{-\infty}$ is $\bigcap_{t \in G} t \cdot F$. Let $X_{0}$ be the conull set $X \backslash R(P(F) \backslash F)$. Then for each $x \in X_{0} \cap F_{0}$, the set $R(x) \cap F$ is nonempty. Also, since $x \notin F_{-\infty}$, there is a $w \in R(x)$ that is not in $F$. Moreover, for any such $w$ and any $z=F \cap R(x)$, we see that $(z, w) \notin P$. For, if $(z, w) \in P$, then $w \in P(F) \cap X_{0} \subseteq F$. Assuming, as we may, that $P$ satisfies $\left(\mathrm{Q}_{2}\right)$, we conclude that for all $w \in R(x) \backslash F$ and all $z \in R(x) \cap F$, we have $(w, z) \in P$. Hence, for each $x$ such that $R(x) \cap F \neq \varnothing$, and for any fixed $w \in R(x) \backslash F$, there results the inequality

$$
d(z, x)=d(z, w)+d(w, x) \leq d(w, x)<\infty
$$

which is valid for all $z \in R(x) \cap F$. This shows that for $x \in X_{0} \cap$ $F_{0}, g(x)$ is well-defined and finite. To see that $g$ is Borel, let $\left\{\varphi_{k}\right\}$ be the sequence of functions defined in Lemma 2.1 and for $n=$ $1,2,3, \ldots, \infty$, let $X_{n}=\{x \in X|| R(x) \mid=n\}$. Then for $t \in \mathbf{R}$,

$$
\begin{array}{r}
g^{-1}(-\infty, t]=\bigcap_{1 \leq n \leq \infty}\left\{x \in F_{0} \cap X_{n} \mid d(y, x) \leq t \text {, for every } y \in F \cap R(x)\right\} \\
=\bigcap_{1 \leq n \leq \infty} \bigcap_{1 \leq k \leq n}\left\{x \in F_{0} \cap X_{n} \mid d\left(\varphi_{k}(x), x\right) \leq t \text { whenever } \varphi_{k}(x) \in F\right\} \\
=\bigcap_{1 \leq n \leq \infty} \bigcap_{1 \leq k \leq n}\left\{x \in F_{0} \cap X_{n} \mid \text { either } \varphi_{k}(x) \notin F \text { or } d\left(\varphi_{k}(x), x\right) \leq t\right\} \\
=\bigcap_{1 \leq n \leq \infty} \bigcap_{1 \leq k \leq n}\left\{x \in F_{0} \cap X_{n} \mid\left(\varphi_{k}(x), x\right) \in[(X \backslash F) \times X]\right. \\
\left.\cup d^{-1}(-\infty, t]\right\} .
\end{array}
$$

Since the terms in this intersection are all Borel, it follows that $g$ is a Borel function. To see that $d=\delta g$ on $R \cap\left(\left(X_{0} \cap F\right) \times\left(X_{0} \cap F\right)\right)$, 
fix $(x, y)$ in this set and observe that for all $z, w \in F \cap R(x)=$ $F \cap R(y), d(z, y) \leq d(w, y)$ if and only if $d(z, x) \leq d(w, x)$ because $d(z, x)=d(z, y)+d(y, x)$ and $d(w, x)=d(w, y)+d(y, x)$. Thus

$$
\begin{aligned}
g(y)-g(x)= & \sup \{d(z, y) \mid z \in F \cap R(y)\} \\
& -\sup \{d(z, x) \mid z \in F \cap R(x)\} \\
= & \sup \{d(z, x)-d(z, x) \mid z \in F \cap R(y)\}=d(x, y) .
\end{aligned}
$$

This completes the proof.

While the correspondence between cocycles and $P$ 's giving rise to analytic algebras is not one to one, the following proposition shows, in a sense, that "the $P$ 's are separated by cohomology". It is a key ingredient in the proof of Theorem 4.9, below.

Proposition 3.9. If $d_{1} \in B^{1}(R, \mathbf{R})$, if $d_{2} \in Z^{1}(R, \mathbf{R})$, and if $P\left(d_{1}\right)=$ $P\left(d_{2}\right)$ a.e. $\nu$, then $d_{2} \in B^{1}(R, \mathbf{R})$.

Proof. Suppose $h$ is a real-valued Borel function on $X$ that satisfies $d_{1}=\delta h$, a.e. $\nu$. Let $\left\{r_{i}\right\}_{i=1}^{\infty}$ be an enumeration of $\mathbf{Q}$ and let $F_{i}=\{x \in$ $X \mid h(x) \geq r_{i}$. Then $F_{i}$ is an increasing subset of $X$ with respect to $P\left(d_{1}\right)$. Since $P\left(d_{1}\right)=P\left(d_{2}\right)$ a.e. $\nu$, each $F_{i}$ is increasing with respect to $P\left(d_{2}\right)$. Using $d_{2}$ we may apply Lemma 3.8 to each $F_{i}$ to find a Borel function $g_{i}$, defined a.e. $\nu$ on $\tilde{F}_{i}:=\left\{x \in X \mid R(x) \cap F_{i}, R(x) \cap\left(X \backslash F_{i}\right) \neq\right.$ $\varnothing\}$, such that

$$
d_{2}(x, y)=g_{i}(y)-g_{i}(x)
$$

for $\nu$-almost all $(x, y)$ in $R \cap\left(\tilde{F}_{i} \times \tilde{F}_{i}\right)$. Since $\mathbf{Q}$ is dense in $\mathbf{R}$, we have $\bigcup_{i=1}^{\infty} \tilde{F}_{i}=X$. Set $K_{1}=\tilde{F}_{1}, K_{2}=\tilde{F}_{2} \cap\left(X \backslash \tilde{F}_{1}\right)$, etc. to arrive at a sequence of pairwise disjoint invariant sets with $\bigcup_{i=1}^{\infty} K_{i}=X$. If we define $g$ to be $g_{i}$ on $K_{i}$, we obtain a well defined function $g$ on $X$ such that $d_{2}=\delta g$ a.e. $\nu$.

4. Periodic flows. A special subclass of the class of analytic algebras are those for which the flow is periodic. Such analytic algebras are the most tractable, and the next proposition identifies the $P$ 's in $\mathfrak{P}$ that correspond to them. If $\alpha$ is periodic, we shall write $H^{\infty}(\alpha, \mathbf{T})$ instead of $H^{\infty}(\alpha)$. This is because a periodic flow may be viewed as a $\sigma$ weakly continuous representation of the unit circle $\mathbf{T}$ as a group of automorphisms on $M$. We will see in this section how periodic flows can be used to construct algebras of the form $\mathfrak{T}(P)$ with $P \notin \mathfrak{P}(a)$ and how they relate to the general problem of deciding when $\mathfrak{P} \neq \mathfrak{P}(a)$. 
THEOREM 4.1. For $P \in \mathfrak{P}$, the following assertions are equivalent:

(1) $\mathfrak{T}(P)=H^{\infty}(\alpha, \mathbf{T})$ for some periodic flow $\alpha$.

(2) For $\nu$-almost all $(x, y) \in P$, the set $\{z \in X \mid(x, z) \in P \backslash \Delta,(z, y) \in$ $P\}$ is finite; i.e., the interval $(x, y]$ is finite.

(3) If $(P \backslash \Delta)^{n}$ denotes the $n$-fold composition $(P \backslash \Delta) \circ \cdots \circ(P \backslash \Delta)$, then $\nu\left(\bigcap_{n \geq 1}(P \backslash \Delta)^{n}\right)=0$.

(4) There is a partial Borel isomorphism $\varphi$ such that $\Gamma(\varphi) \subseteq P \backslash \Delta$ and $P \backslash \Delta=\bigcup_{n=1}^{\infty} \Gamma\left(\varphi^{n}\right)$ a.e. $\nu$.

Proof. (1) implies (3). Since $\alpha$ is an action of $\mathbf{T}$, there is a $d \in$ $Z^{1}(R, Z)$ such that $P=P(d)$ a.e. $\nu$. Hence $P \backslash \Delta=\{(x, y) \mid d(x, y) \geq 1\}$ and $(P \backslash \Delta)^{n} \subseteq\{(x, y) \mid d(x, y) \geq n\}$. This proves (3).

(3) implies (4). Let $B=(P \backslash \Delta) \backslash(P \backslash \Delta)^{2}$. Then both $\pi_{r} \mid B$ and $\pi_{l} \mid B$ are one-to-one. (Recall $\pi_{l}(x, y)=x, \pi_{r}(x, y)=y$.) Indeed, if $(x, y),(x, z) \in B$, then either $(y, z) \in P \backslash \Delta,(z, y) \in P \backslash \Delta$, or $y=z$. (We're assuming $P$ satisfies $\left(\mathrm{Q}_{2}\right)$, as we may by Lemma 3.1 of [MSS].) If $(y, z) \in P \backslash \Delta$, then $(x, z) \in(P \backslash \Delta)^{2}$ and if $(z, y) \in P \backslash \Delta$, then $(x, y) \in(P \backslash \Delta)^{2}$. Hence the only way for $(x, y)$ and $(x, z)$ to be in $B$ is for $y$ to equal $z$; i.e., $\pi_{l}$ is injective. The injectivity of $\pi_{r}$ is proved similarly. Thus $B$ is the graph of a partial Borel isomorphism. For $n \geq 1$, we have $\Gamma\left(\varphi^{n}\right)=(P \backslash \Delta)^{n} \backslash(P \backslash \Delta)^{n+1}$ and so $P \backslash \Delta=\left(\bigcup_{n \geq 1} \Gamma\left(\varphi^{n}\right)\right) \cup\left(\bigcap_{n \geq 1}(P \backslash \Delta)^{n}\right)$. This shows that (4) follows from (3).

(4) implies (2). Let $\varphi$ be a partial Borel isomorphism satisfying the equation $P \backslash \Delta=\bigcup_{n=1}^{\infty} \Gamma\left(\varphi^{n}\right)$ a.e. $\nu$. Certainly $(x, x]$ is finite. So it suffices to prove that $(x, y]$ is finite for all $(x, y) \in \bigcup_{n=1}^{\infty} \Gamma\left(\varphi^{n}\right)$. But for $(x, y) \in \Gamma\left(\varphi^{n}\right)$, the interval $(x, y]$ is the finite ordered set $\left\{\varphi(x), \varphi^{2}(x), \ldots, \varphi^{n}(x)\right\}$.

(2) implies (1). Simply set

$$
\begin{aligned}
d(x, y)= & |\{z \mid(x, z) \in P \backslash \Delta,(z, y) \in P\}| \\
& -|\{z \mid(y, z) \in P \backslash \Delta,(z, x) \in P\}| .
\end{aligned}
$$

Then $d(x, y)$ is the number of elements in $(x, y]$, if $(x, y) \in P$, and it is $-|(y, x]|$, if $(y, x) \in P$. It is evident that $d$ is a cocycle and $P(d)$ differs from $P$ by a null set.

REMARK 4.2. Let $P$ and $\varphi$ be as in the previous theorem and let $d$ be the cocycle defined in the last part of the proof. Then, except possibly for a $\nu$-null set, $R=\bigcup_{n=-\infty}^{\infty} \Gamma\left(\varphi^{n}\right)$, where $\varphi^{0}$ is the identity. 
So for all $x$ in a $\mu$-conull set, $R(x)=\left\{\varphi^{n}(x) \mid n \in \mathbf{Z}\right.$ and $\varphi^{n}(x)$ is defined\}. Moreover, we see that $d \mid \Gamma\left(\varphi^{n}\right)=n$. This is evident from the proof. It follows that the operator $L_{F\left(\varphi^{n}\right)}$ lies in the spectral subspace $M^{\alpha}(n)=\left\{T \in M \mid \alpha_{t}(T)=e^{\text {int }} T\right.$, for all $\left.t \in \mathbf{R}\right\}$. (Recall that for $T \in M, a\left(\alpha_{t}((T))(x, y)=e^{i t d(x, y)} a(T)\right)(x, y)$.) In fact,

$$
\begin{aligned}
M^{\alpha}(n) & =\left\{T \in M \mid a(T) \text { is supported on } d^{-1}(n)\right\} \\
& =\left\{T \in M \mid \operatorname{supp}(a(T)) \subseteq \Gamma\left(\varphi^{n}\right)\right\}
\end{aligned}
$$

A simple calculation shows that $T=E\left(T L_{F\left(\varphi^{-n}\right)}\right) L_{F\left(\varphi^{n}\right)}$ for all $T \in$ $M^{\alpha}(n)$. Hence $M^{\alpha}(n)=A L_{F\left(\varphi^{n}\right)}=A\left(L_{F(\varphi)}\right)^{n}$ for all $n \geq 0$. Since the $\sigma$-weakly closed linear span of $\bigcup_{n=0}^{\infty} M^{\alpha}(n)$ is $\mathfrak{T}(P)$, it follows that $\mathfrak{T}(P)$ is the $\sigma$-weakly closed algebra generated by $A$ and $L_{F(\varphi)}$.

It follows from [FM2] that $M$ is a hyperfinite $\mathrm{II}_{1}$ factor if and only if $R$ is the orbit equivalence relation determined by a single invertible, ergodic, measure preserving transformation, say, $\tau$. In this case we may choose $s \equiv 1$.

Theorem 4.1, then, yields an easily checked sufficient condition for deciding when a $d \in Z^{1}(R, \mathbf{R})$, having the property that $P(d) \in \mathfrak{P}$, satisfies $\mathfrak{T}(P(d))=H^{\infty}(\alpha, \mathbf{T})$ for a periodic flow $\alpha$. It turns out, because of Corollaries 11.2 and 11.3 of [Sc], that the following dichotomy holds: either $\lim _{|n| \rightarrow \infty}\left|d\left(x, \tau^{n}(x)\right)\right|=\infty$ a.e. $\mu$ or $\varliminf_{|n| \rightarrow \infty}\left|d\left(x, \tau^{n}(x)\right)\right|$ $=0$. In the first case $d$ is called transient; in the second, $d$ is called recurrent. These terms are usually defined differently, but thanks to the indicated corollaries, we can define them as we did.

COROLLARY 4.3. Let $R$ be the orbit equivalence relation determined by an ergodic measure preserving transformation $\tau$ and let $d \in Z^{1}(R, \mathbf{R})$ be such that $P(d) \in \mathfrak{P}$. Then $\mathfrak{T}(P(d))$ is of the form $H^{\infty}(\alpha, \mathbf{T})$ for $a$ periodic flow $\alpha$ if and only if $d$ is transient. Moreover, if $c(x)$ is defined to be $d(x, \tau(x))$ and if $c$ is integrable, then this happens if and only if $\int c(x) d \mu(x) \neq 0$.

Proof. If $d$ is transient, then it is easily checked that

$$
\lim _{n \rightarrow \infty} d\left(x, \tau^{n}(x)\right)=\infty \text { or }-\infty \text { a.e. } \mu .
$$

Without loss of generality, we assume that this limit is $\infty$. Then $\lim _{n \rightarrow-\infty} d\left(x, \tau^{n}(x)\right)=-\infty$ a.e. $\mu$. Fix $(x, y) \in P(d)$ with the property that $\lim _{n \rightarrow \infty} d\left(x, \tau^{n}(x)\right)=\infty$. Then we can find an $N$ such that for $n>N, d\left(x, \tau^{n} x\right)>d(x, y)$ and for $n<-N, d\left(x, \tau^{n} x\right) \leq 0$. It follows 
that for all $n$ with $|n|>N, \tau^{n}(x) \notin(x, y]$. For, if $\tau^{n}(x) \in(x, y]$, then $\left(x, \tau^{n}(x)\right) \in P$, implying that $n \geq-N$, and then

$$
d(x, y)=d\left(x, \tau^{n}(x)\right)+d\left(\tau^{n}(x), y\right) \geq d\left(x, \tau^{n}(x)\right),
$$

implying that $n \leq N$. Thus $(x, y]$ is finite. Now apply Theorem 4.1. If $d$ is not transient, it is recurrent; i.e., $\underline{\lim }_{|n| \rightarrow \infty}\left|d\left(x, \tau^{n}(x)\right)\right|=0$ a.e. $\mu$. Hence, for almost all $(x, y)$ in $P \backslash \Delta$, we can find a sequence of positive integers $\left\{n_{k}\right\}_{k=1}^{\infty}$ (depending on $x$ ) such that $d\left(x, \tau^{n_{k}}(x)\right)>0$ for each $k$ and $\sum_{k=1}^{\infty} d\left(x, \tau^{n_{k}}(x)\right) \leq d(x, y)$. It follows that $\left\{\tau^{n_{k}}(x)\right\}_{k=1}^{\infty} \subseteq$ $(x, y]$; i.e., for almost all $(x, y)$ in $P \backslash \Delta$, the interval $(x, y]$ is infinite. Now apply Theorem 4.1. For the last assertion, simply appeal to Theorem 11.4 of $[\mathbf{S c}]$.

Corollary 4.4. Suppose that $P \in \mathfrak{P}$ and that statements (1)-(4) of Theorem 4.1 are satisfied. Then $M=M(R, s)$ is hyperfinite, so that we may assume that $s \equiv 1$, and we may write $X$ as the disjoint union of two invariant Borel sets $Y$ and $Z$ satisfying the following conditions. (Of course either $Y$ or $Z$ may be empty.)

(1) $M(R \cap(Y \times Y))$ is isomorphic to the crossed product $L^{\infty}(Y) \rtimes_{\varphi} \mathbf{Z}$, where $\varphi$ is the restriction of the partial Borel isomorphism in Theorem 4.1(4) to $Y$, and $\mathfrak{T}(P \cap(Y \times Y))$ is isomorphic to the analytic crossed product $L^{\infty}(Y) \rtimes_{\varphi} \mathbf{Z}_{+}$, which is, by definition, $H^{\infty}(\hat{\varphi})$ where $\hat{\varphi}$ is the dual action of $\varphi$ on $L^{\infty}(Y) \rtimes_{\varphi} \mathbf{Z}$.

(2) The algebra $\mathfrak{T}(P \cap(Z \times Z))$ is a nest subalgebra of $M(R \cap(Z \times Z))$.

Proof. Since, by hypothesis, the fixed point algebra $M^{\alpha}$ of the periodic flow $\alpha$ is $A$, a hyperfinite von Neumann algebra, $M$ is hyperfinite (see [S1, last remark]). Define $Z$ to be $\{x \in X \mid$ there is an $n \in \mathbf{Z}$ such that $\left.x \notin d\left(\varphi^{n}\right)\right\}$. Then it is easy to see that $Z$ is invariant and Borel, and that $Z=\{x \in X \mid$ either $[x, \infty)$ or $(-\infty, x]$ is finite $\}$, where $[x, \infty)=\{y \in X \mid(x, y) \in P\}$ while $(-\infty, x]=\{y \in X \mid(y, x) \in P\}$. Set $Y=X \backslash Z$. Since $\Gamma(\varphi)=(P \backslash \Delta) \backslash(P \backslash \Delta)^{2}, \varphi$ is defined on all of $Y$ and maps $Y$ onto all of $Y$. Thus $\varphi \mid Y$ is a Borel isomorphism on $Y$ and $R \cap(Y \times Y)$ is just the orbit equivalence relation determined by $\varphi \mid Y$. Moreover, by definition, $\varphi$ is freely acting on $Y$. Hence $M(R \cap(Y \times Y))$ may be identified with $L^{\infty}(Y) \rtimes_{\varphi} \mathbf{Z}$. Also, since

$$
\begin{aligned}
P \cap(Y \times Y) & =\left\{\left(x, \varphi^{n}(x)\right) \mid x \in Y, n \geq 0\right\} \\
& =\{(x, y) \in R \cap(Y \times Y) \mid d(x, y) \geq 0\},
\end{aligned}
$$

where $d$ is the cocycle in Theorem 4.1(4). Restricting $d$ to $R \cap(Y \times Y)$ yields a cocycle and automorphism group $\beta$ such that

$$
\beta_{t}\left(L_{F\left(\varphi^{n}\right)}\right)=\left(e^{i t n}\right) L_{F\left(\varphi^{n}\right)}, \quad n \in \mathbf{Z}, t \in \mathbf{R} .
$$


It is thus evident that $\beta=\hat{\varphi}$ and $\mathfrak{T}(P \cap(Y \times Y))=H^{\infty}(\beta)$.

It remains to show that $\mathfrak{T}(P \cap(Z \times Z))$ is a nest algebra. Write $Z_{1}=\left\{x \in X \mid\right.$ for some $\left.n>0, x \notin d\left(\varphi^{n}\right)\right\}$. Then $Z_{1}$ is an invariant Borel set and $Z_{1}=\{x \in X \mid[x, \infty)$ is finite $\}$. Set $Z_{2}=Z \backslash Z_{1}$. Then $Z_{2} \subseteq\{x \in X \mid(-\infty, x]$ is finite $\}$. Define the real-valued function $g$ on $Z$ by the formula

$$
g(x)=\left\{\begin{array}{cc}
-|[x, \infty)|, & x \in Z_{1}, \\
|(-\infty, x]|, & x \in Z_{2} .
\end{array}\right.
$$

It is straightforward to check that for $(x, y)$ in $R \cap(Z \times Z)=(R \cap$ $\left.\left(Z_{1} \times Z_{1}\right)\right) \cup\left(R \cap\left(Z_{2} \times Z_{2}\right)\right), d(x, y)=g(y)-g(x)$, where $d(x, y)=$ $|(x, y]|-|(y, x]|$, the cocycle that appears in Theorem 4.1(4). Now apply Corollary 3.4 .

REMARK 4.5. Theorem 4.1 says that for $P \in \mathfrak{P}, \mathfrak{T}(P)=H^{\infty}(\alpha, \mathrm{T})$ for a periodic flow $\alpha$ if and only if almost every equivalence class $R(x)$ is order isomorphic to a subset of $\mathbf{Z}$. Suppose next that $\mathfrak{T}(P), P \in \mathfrak{P}$, is contained in an algebra of the form $H^{\infty}(\alpha, \mathbf{T})$ and let $d \in Z^{1}(R, \mathbf{Z})$ be a cocycle that implements $\alpha$. Then $\Delta \subseteq d^{-1}(0)$ and $\mathfrak{T}(P)$ is properly contained in $H^{\infty}(\alpha, \mathbf{T})$ if and only if $\Delta$ is properly contained in $d^{-1}(0)$. If we restrict $d$ to $R(x) \times R(x)$, the values that $d$ takes on there may be used to partition $R(x)$ into "blocks": $y, z \in R(x)$ belong to the same block if and only if $d(y, z)=0$. To say the same thing differently, $y, z \in R(x)$ belong to the same block if and only if for all $u \in R(x), d(u, y)=d(u, z)$. For $z \in R(x)$, we write $R_{0}(z)$ for the block it determines; i.e., $R_{0}(z)=\{y \in R(x)=R(z) \mid d(z, y)=0\}$. Of course $d$ induces an ordering on the set of blocks in $R(x)$ and this ordering is order isomorphic to a subset of $\mathbf{Z}$. That is, $R_{0}(u) \leq R_{0}(v)$ if and only if $d(u, v) \geq 0$. The orderings that $P$ induces inside each block may, a priori, be quite arbitrary. However, as we shall see in our next proposition, if $P \in \mathfrak{P}(a)$ and if the number of blocks in $R(x)$ is greater than one for almost all $x \in X$, then the order within the blocks is such that $\mathfrak{T}(P) \cap M^{\alpha}$ is a nest subalgebra of $M^{\alpha}$; i.e., the triangular subalgebra $\mathfrak{T}\left(P \cap d^{-1}(0)\right)$ is a nest subalgebra of $M^{\alpha}=\mathfrak{T}\left(d^{-1}(0)\right)$.

Proposition 4.6. Suppose that $P \in \mathfrak{P}$ and that

$$
\mathfrak{T}=\mathfrak{T}(P) \subseteq H^{\infty}(\alpha, \mathbf{T})
$$

for some periodic flow $\alpha$. Suppose also that there is no nonzero projection $L_{e} \in A$ such that $M L_{e}=M^{\alpha} L_{e}$. Then $P$ lies in $\mathfrak{P}(a)$ if and only if $\mathfrak{T} \cap M^{\alpha}$ is a nest subalgebra of $M^{\alpha}$. 
Proof. Let $d$ be the cocycle implementing $\alpha$, let $L_{e}$ be a projection in $A$, and let $X_{0}$ be the subset of $X$ determined by $L_{e}$; i.e., $e=1_{X_{0}}$. Then $M L_{e}=\left\{T \in M \mid \operatorname{supp} a(T) \subseteq R \cap\left(X \times X_{0}\right)\right\}$ while $M^{\alpha} L_{e}=$ $\left\{T \in M \mid \operatorname{supp} a(T) \subseteq d^{-1}(0) \cap\left(X \times X_{0}\right)\right\}$. Our hypothesis on $d$, then, asserts that there is no subset $X_{0} \subseteq X$ with $\mu\left(X_{0}\right) \neq 0$ and $\mu\left(X \backslash X_{0}\right) \neq 0$ such that $d \mid\left(X \times X_{0}\right) \cap R=0$.

Suppose first that $P \in \mathfrak{P}(a)$ and write $P=P\left(d_{0}\right)$ for a suitable $d_{0} \in Z^{1}(R, \mathbf{R})$. Set $X_{1}=\{x \in X \mid$ there exists a $y \in X$ with $(x, y) \in P$ such that $d(x, y)>0\}$ and set $X_{2}=\{x \in X \mid$ there exists $y \in X$ with $(y, x) \in P$, and $d(y, x)>0\}$. In the notation of Remark 4.5, $X_{1}$ consists of those $x \in X$ such that the block $R_{0}(x)$ in $R(x)$ is not the largest among the blocks in $R(x)$; likewise, $X_{2}$ consists of those $x \in X$ such that the block $R_{0}(x)$ in $R(x)$ is not the smallest. If $X_{0}=X \backslash\left(X_{1} \cup X_{2}\right)$, then it is clear that $d$ vanishes on $\left(X \times X_{0}\right) \cap R$. Hence, by the preceding paragraph, $\mu\left(X_{0}\right)=0$. We assume, without loss of generality, that $X=X_{1} \cup X_{2}$. Define $g$ by the formula

$$
g(x)= \begin{cases}\sup \left\{d_{0}(y, x) \mid d(x, y)>0\right\}, & x \in X_{1}, \\ \inf \left\{d_{0}(y, x) \mid d(y, x)>0\right\}, & x \in X_{2} \backslash X_{1}\end{cases}
$$

To see that $g$ is Borel, note that for $t \in \mathbf{R},\left(g^{-1}(-\infty, t]\right) \cap X_{1}=\left\{x \in X_{1} \mid\right.$ for all $y \in R(x)$, either $d(x, y) \leq 0$ or $\left.d_{0}(y, x) \leq t\right\}=\bigcap_{n=1}^{\infty}\left\{x \in X_{1} \mid\right.$ either $d\left(x, \varphi_{n}(x)\right) \leq 0$ or $\left.d_{0}\left(\varphi_{n}(x), x\right) \leq t\right\}$, where the $\varphi_{n}$ 's are as in Lemma 2.1. Thus the restriction $\left.g\right|_{X_{1}}$ is Borel. A similar argument shows that $\left.g\right|_{X_{2} \backslash X_{1}}$ is Borel. Write $R_{0}=d^{-1}(0)$. Then $R_{0}$ is an equivalence relation contained in $R$ and $M^{\alpha}$ may be identified with $M\left(R_{0}, s\right)$. (Actually, we have been a little cavalier with null sets; we might have to modify $R_{0}$ slightly to get an equivalence relation, but that is o.k. by Lemma 3.1 of [MSS].) Set $P_{0}=P \cap R_{0}=P\left(d_{0}\right) \cap R_{0}$. Then $\mathfrak{T} \cap M^{\alpha}=\mathfrak{T}\left(P_{0}\right)$. We claim that $P_{0}=\left\{(x, y) \in R_{0} \mid g(x) \leq g(y)\right\}$. By Corollary 3.4, this will show that $\mathfrak{T} \cap M^{\alpha}$ is a nest algebra in $M^{\alpha}$. Observe that since $d$ vanishes on $R_{0}$, each of the sets $X_{1}$ and $X_{2}$ are invariant under $R_{0}$. So

$$
R_{0}=\left(R_{0} \cap\left(X_{1} \times X_{1}\right)\right) \cup\left(R_{0} \cap\left(\left(X_{2} \backslash X_{1}\right) \times\left(X_{2} \backslash X_{1}\right)\right)\right) .
$$

We therefore prove that for $(x, y) \in R_{0} \cap\left(X_{1} \times X_{1}\right),(x, y) \in P$ if and only if $g(y) \geq g(x)$; the argument for $R_{0} \cap\left(\left(X_{2} \backslash X_{1}\right) \times\left(X_{2} \backslash X_{1}\right)\right)$ is similar and will be omitted. So let $(x, y) \in R_{0} \cap\left(X_{1} \times X_{1}\right)$ and note 
that since $d(x, y)=0$,

$$
\begin{aligned}
g(y) & =\sup \left\{d_{0}(z, y) \mid d(y, z)>0\right\} \\
& =\sup \left\{d_{0}(z, x)+d_{0}(x, y) \mid d(y, x)+d(x, z)>0\right\} \\
& =d_{0}(x, y)+\sup \left\{d_{0}(z, x) \mid d(x, z)>0\right\} \\
& =d_{0}(x, y)+g(x) .
\end{aligned}
$$

Thus for $(x, y) \in R_{0} \cap\left(X_{1} \times X_{1}\right), g(y) \geq g(x)$ if and only if $d_{0}(x, y) \geq$ 0 ; i.e., if and only if $(x, y) \in P\left(d_{0}\right)=P$.

For the converse implication, suppose that $\mathfrak{T} \cap M^{\alpha}$ is a nest subalgebra of $M^{\alpha}$. Then there is a real-valued Borel function $g$ on $X$ such that for $(x, y)$ in $R_{0}=d^{-1}(0), g(x) \leq g(y)$ if and only if $(x, y) \in P$. By replacing $g$ with $(1 / \pi) \arctan (g)$, we may assume that $|g|<\frac{1}{2}$. Set $d_{0}(x, y)=d(x, y)+g(y)-g(x)$. Then since $d \in Z^{1}(R, \mathbf{Z}) \subseteq Z^{1}(R, \mathbf{R})$, we see that $d_{0} \in Z^{1}(R, \mathbf{R})$. To see that $P=P\left(d_{0}\right)$, suppose that $(x, y) \in P$. Then $d(x, y) \geq 0$, since $\mathfrak{T}(P) \subseteq H^{\infty}(\alpha, \mathbf{T})=\mathfrak{T}(P(d))$. If $d(x, y)=0$, then $(x, y) \in R_{0} \cap P$ and so $d_{0}(x, y)=0+g(y)-g(x) \geq 0$; i.e., $(x, y) \in P\left(d_{0}\right)$. If $d(x, y)>0$, then since $d$ is Z Z-valued, $d(x, y) \geq$ 1 , and $d_{0}(x, y) \geq 1+g(y)-g(x) \geq 0$, since $|g|<\frac{1}{2}$. Thus $P \subseteq P\left(d_{0}\right)$. To show the reverse inclusion, it suffices to show that $P\left(d_{0}\right) \in \mathfrak{P}$. But if $(x, y) \in P\left(d_{0}\right) \cap \theta\left(P\left(d_{0}\right)\right), d_{0}(x, y)=0$. So $d(x, y)=0$; i.e., $(x, y) \in R_{0}$, and the equation $d_{0}(x, y)=0$ implies $g(y)=g(x)$. Thus $x=y$.

Suppose $L_{e}$ is a projection in $A$ different from 0 and $I$, and let $Y$ be the corresponding set in $X$. For $P \in \mathfrak{P}$ and $\mathfrak{T}=\mathfrak{T}(P)$, we write

$$
\mathfrak{T}_{e}=L_{e} \mathfrak{T} L_{e}+L_{e}^{\perp} \mathfrak{T} L_{e}^{\perp}+L_{e} M L_{e}^{\perp}
$$

and

$$
P_{Y}=(P \cap(Y \times Y)) \cup(P \cap((X \backslash Y) \times(X \backslash Y))) \cup(R \cap(Y \times(X \backslash Y)))
$$

where $L_{e}^{\perp}=I-L_{e}$. It is clear that $\mathfrak{T}_{e}=\mathfrak{T}\left(P_{Y}\right), P_{Y} \in \mathfrak{P}$, and so $\mathfrak{T}_{e}$ is a maximal subdiagonal subalgebra of $M$ with respect to $E$.

LEMMA 4.7. Suppose that $P, L_{e}$, and $Y$ are as above and assume that $R(Y)=X=R(X \backslash Y)$ a.e. $\nu$. Then the following assertions are equivalent:

(1) $P_{Y} \in \mathfrak{P}(a)$.

(2) $L_{e} \mathfrak{T} L_{e}+L_{e}^{\perp} \mathfrak{T} L_{e}^{\perp}$ is a nest subalgebra of $L_{e} M L_{e}+L_{e}^{\perp} M L_{e}^{\perp}$.

(3) $L_{e} \mathfrak{T} L_{e}$ and $L_{e}^{\perp} \mathfrak{T} L_{e}^{\perp}$ are nest subalgebras of $L_{e} M L_{e}$ and $L_{e}^{\perp} M L_{e}^{\perp}$, respectively.

Proof. (1) and (2) are equivalent. Clearly $\mathfrak{T}_{e}$ is contained in $M \cap$ $\operatorname{alg}\left\{0, L_{e}, I\right\}$. By Theorem 4.2.3 of [LM], this second algebra is of the 
form $H^{\infty}(\alpha, \mathbf{T})$ for a periodic flow $\alpha$ on $M$. Since $M^{\alpha}=L_{e} M L_{e}+$ $L_{e}^{\perp} M L_{e}^{\perp}$ and $\mathfrak{T}_{e} \cap M^{\alpha}=L_{e} \mathfrak{T} L_{e}+L^{\perp} \mathfrak{T} L_{e}^{\perp}$, it suffices, by Proposition 4.6, to show that for no projection $L_{f} \in A$, different from 0 , is the equation $M L_{f}=M^{\alpha} L_{f}$ satisfied. However, if $M L_{f}=M^{\alpha} L_{f}$, then $L_{e} M L_{e} L_{f}=L_{e} M L_{f}$ and $L_{e}^{\perp} M L_{e}^{\perp} L_{f}=L_{e}^{\perp} M L_{f}$. The assumption that $R(Y)=X$ implies that $L_{e} M L_{p} \neq 0$ for every nonzero projection $L_{p} \in A$. But the equation $L_{e} M L_{e} L_{f}=L_{e} M L_{f}$ implies that $L_{e} M\left(L_{f}-L_{f} L_{e}\right)=0$. Hence $L_{f} \leq L_{e}$, and it follows that $L_{e}^{\perp} M L_{f}=L_{e}^{\perp} M L_{e}^{\perp} L_{f}=0$. Using the fact that $R(X \backslash Y)=X$, we see that $L_{f} \leq L_{e}^{\perp}$. Thus $L_{f}=0$.

(2) and (3) are equivalent. Suppose $L_{e} \mathfrak{T} L_{e}+L_{e}^{\perp} \mathfrak{T} L_{e}^{\perp}$ is a nest subalgebra of $L_{e} M L_{e}+L_{e}^{\perp} M L_{e}^{\perp}$, say the former is the intersection of the latter with $\operatorname{alg}\left\{L_{q_{t}}\right\}$ for a totally ordered family of projections $\left\{L_{q_{t}}\right\}$ contained in the latter. Then, since $A \subseteq L_{e} M L_{e}+L_{e}^{\perp} M L_{e}^{\perp}$, it follows that each $L_{q_{t}} \in A$, and so $L_{e} \mathfrak{T} L_{e}=L_{e} M L_{e} \cap \operatorname{alg}\left\{L_{q_{t}} L_{e}\right\}$ while $L_{e}^{\perp} \mathfrak{T} L_{e}^{\perp}=L_{e}^{\perp} M L_{e}^{\perp} \cap \operatorname{alg}\left\{L_{q_{t}} L_{e}^{\perp}\right\}$; i.e., (3) is satisfied. Conversely, if $L_{e} \mathfrak{T} L_{e}=L_{e} M L_{e} \cap \operatorname{alg}\left\{L_{p_{t}}\right\}$ and $L_{e}^{\perp} \mathfrak{T} L_{e}^{\perp}=L_{e}^{\perp} M L_{e}^{\perp} \cap \operatorname{alg}\left\{L_{q_{t}}\right\}$ where the $L_{p_{t}}$ 's are projections in $L_{e} M L_{e}$ and the $L_{q_{t}}$ 's are projections in $L_{e}^{\perp} M L_{e}^{\perp}$, then the $L_{p_{t}}$ and $L_{q_{t}}$ are subprojections of $L_{e}$ and $L_{e}^{\perp}$, respectively, all belonging to $A$, and $L_{e} \mathfrak{T} L_{e}+L_{e}^{\perp} \mathfrak{T} L_{e}^{\perp}=\left(L_{e} M L_{e}+\right.$ $\left.L_{e}^{\perp} M L_{e}^{\perp}\right) \cap \operatorname{alg}\left\{L_{r_{t}}\right\}$ where $\left\{L_{r_{t}}\right\}=\left\{L_{p_{t}}\right\} \cup\left\{L_{e}+L_{q_{t}}\right)$ is ordered in the obvious way.

We come now to an example that shows that $\mathfrak{P}(a)$ need not equal $\mathfrak{P}$. It is followed by Theorem 4.9 that shows among other things that if $\mathfrak{P}=\mathfrak{P}(a)$ and if $M$ is hyperfinite, then $M$ is type $\mathrm{I}$. This is the main result of the section.

EXAmple 4.8. Let $X$ be the interval $[0,1]$, let $\mu$ be Lebesgue measure, let $\tau$ be an irrational translation, let $R$ be the orbit equivalence relation determined by $\tau$, and let $P=\left\{(x, y) \in R \mid y=\tau^{n}(x), n \geq 0\right\}$. Then $P \in \mathfrak{P}(a)$; in fact $P=P(d)$ where $d\left(x, \tau^{n}(x)\right)=n$. We let $M=M(R, 1)$. Let $Y \subseteq X$ be such that $\mu(Y) \neq 0 \neq \mu(X \backslash Y)$, and let $L_{e}$ be the corresponding projection in $A$. Since $\tau$ is ergodic, $Y$ and $L_{e}$ satisfy the hypotheses of Lemma 4.7. Also, the transformation $\tau_{Y}$ on $Y$ induced by $\tau$ is an ergodic measure preserving transformation on $Y$, and we have $R \cap(Y \times Y)=\left\{\left(x, \tau_{Y}^{n}(x)\right) \mid n \in \mathbf{Z}, x \in Y\right\}$. We shall write $R_{0}$ for $R \cap(Y \times Y)$ and $P_{0}$ for $R_{0} \cap P$. We then have $L_{e} M L_{e}=M\left(R_{0}, 1\right)$ and $L_{e} \mathfrak{T} L_{e}=\mathfrak{T}\left(P_{0}\right)$. Since $\tau_{Y}$ is ergodic and $\left(x, \tau_{Y}^{n}(x)\right) \in P_{0}$ for all $x \in Y$ and $n \geq 0$, there are no $P_{0}$-increasing subsets of $Y$. Hence $\mathfrak{T}\left(P_{0}\right)$ is not a nest subalgebra of $L_{e} M L_{e}$. By Lemma 4.7, then, $P_{Y} \notin \mathfrak{P}(a)$. 
Theorem 4.9. Suppose that $M$ is a factor; so, equivalently, $R$ is ergodic. Consider the following assertions:

(1) $\mathfrak{P}=\mathfrak{P}(a)$.

(2) $\mathfrak{P}=\mathfrak{P}(n)$.

(3) $M$ is type I.

Then (1) and (2) are equivalent, (3) implies (2), and if $M$ is hyperfinite, then (3) is equivalent to (1) and (2).

Proof. It is trivial that (2) implies (1) and the fact that (3) implies (2) was proved in Theorem 3.6.

(1) implies (2). Fix $P \in \mathfrak{P}$ and assume that $P=P(d)$ for some $d \in$ $Z^{1}(R, \mathbf{R})$. Let $Y_{0} \subseteq X$ be any Borel set with $\mu\left(Y_{0}\right) \neq 0 \neq \mu\left(X \backslash Y_{0}\right)$ and let $L_{e}$ be the corresponding projection in $A$. By Lemma 4.7, $L_{e} \mathfrak{T} L_{e}$ and $L_{e}^{\perp} \mathfrak{T} L_{e}^{\perp}$ are nest subalgebras of $L_{e} M L_{e}$ and $L_{e}^{\perp} M L_{e}^{\perp}$, respectively, where $\mathfrak{T}=\mathfrak{T}(P)$. Writing $Y_{1}=X \backslash Y_{0}$, we may find real-valued Borel functions $g_{i}$ on $Y_{i}, i=0,1$, such that

$$
d(x, y)=g_{i}(y)-g_{i}(x), \quad(x, y) \in R \cap\left(Y_{i} \times Y_{i}\right) \backslash N_{i}
$$

where $N_{i}$ is a $\nu$-null set of $R \cap\left(Y_{i} \times Y_{i}\right)$. We will find a constant $c$ such that if $g$ is defined by the formula

$$
g(x)= \begin{cases}g_{1}(x), & x \in Y_{1}, \\ g_{0}(x)+c, & x \in Y_{0},\end{cases}
$$

then $d(x, y)=g(y)-g(x)$ a.e. $\nu$. We begin by paring away some null sets. Let $N=N_{0} \cup N_{1}$ and $Y_{i}^{\prime}=Y_{i} \backslash \pi_{r}(N)$. (Note that $\mu\left(\pi_{r}(N)\right)=0$.) Also, let $B=\pi_{l}\left(R \cap\left(Y_{0} \times Y_{1}^{\prime}\right)\right)$. Then $B$ is a subset of $Y_{0}$ and is invariant under $R \cap\left(Y_{0} \times Y_{0}\right)$. Since $R \cap\left(Y_{0} \times Y_{0}\right)$ is ergodic, by Proposition 3.5 of [FM1], we conclude that $B$ is a conull subset of $Y_{0}$. Therefore, so is $Y_{0}^{\prime \prime}:=Y_{0}^{\prime} \cap B$. For $y \in Y_{0}^{\prime \prime}$, there is an $x \in Y_{1}^{\prime}$ such that $x \sim y$. For such a pair $(x, y)$, let $f(x, y)=d(x, y)+g_{1}(x)-g_{0}(y)$. If $z$ is another element in $Y_{1}^{\prime} \cap R(y)$, then

$$
\begin{aligned}
f(z, y) & =d(z, y)+g_{1}(z)-g_{0}(y) \\
& =d(z, x)+d(x, y)+g_{1}(z)-g_{0}(y) \\
& =g_{1}(x)-g_{1}(z)+d(x, y)+g_{1}(z)-g_{0}(y)=f(x, y) .
\end{aligned}
$$

Thus $f$ is independent of the choice of $x \in Y_{1}^{\prime} \cap R(y)$ and we may write $f(y)$ for $f(x, y)$. Evidently, $f$ is a Borel function on $Y_{0}^{\prime \prime}$. Now choose $y, w \in Y_{0}^{\prime \prime}, y \sim w$, and choose $x \in Y_{1}^{\prime} \cap R(y)=Y_{1}^{\prime} \cap R(w)$. 
Then

$$
\begin{aligned}
f(y) & =f(x, y)=d(x, y)+g_{1}(x)-g_{0}(y) \\
& =d(x, w)+d(w, y)+g_{1}(x)-g_{0}(y) \\
& =d(x, w)+g_{0}(y)-g_{0}(w)+g_{1}(x)-g_{0}(y)=f(x, w)=f(w) .
\end{aligned}
$$

Thus $f$ is invariant, and by ergodicity, there is a $c \in \mathbf{R}$ and a conull set $Y \subseteq Y_{0}^{\prime \prime} \subseteq Y_{0}$ such that $f \equiv c$ on $Y$. With this $c$, define $g$ on $X$ by equation (4.1). By construction, $d(x, y)=g(y)-g(x)$ for almost all $(x, y) \in R \cap\left(Y_{0} \times Y_{0}\right)$ and for almost all $(x, y) \in R \cap\left(Y_{1} \times Y_{1}\right)$. So to prove that $d(x, y)=g(y)-g(x)$ a.e. on all of $R$, it suffices to check that $d(x, y)=g(y)-g(x)$ a.e. on $R \cap\left(Y_{1} \times Y_{0}\right)$. (This is because $R \cap\left(Y_{0} \times Y_{1}\right)=\theta\left(R \cap\left(Y_{1} \times Y_{0}\right)\right)$.) Since $Y_{1}^{\prime}$ and $Y$ are conull sets of $Y_{1}$ and $Y_{0}$, respectively, it suffices to assume that $(x, y) \in Y_{1}^{\prime} \times Y$. But then, $c=f(x, y)=d(x, y)+g_{1}(x)-g_{0}(y)$, so $d(x, y)=c+g_{0}(y)-$ $g_{1}(x)=g(y)-g(x)$ by definition. Thus $d(x, y)=g(y)-g(x)$ a.e. $\nu$.

(2) implies (3) (assuming $M$ is hyperfinite). Since $M$ is hyperfinite, there is a transformation $\tau$ on $X$ such that $R=\left\{\left(x, \tau^{n}(x)\right) \mid n \in \mathbf{Z}\right\}$. By ergodicity, we may assume $|R(x)|$ is constant. If this constant is finite, $R$ is type I. So we may assume $|R(x)|=\infty$ a.e. $\mu$. As a consequence, we see that $\tau$ is freely acting. Let $P=\left\{\left(x, \tau^{n}(x)\right) \mid n \geq 0\right\}$. So $P=P(d)$ where $d\left(x, \tau^{n}(x)\right)=n$. By hypothesis (2) and Corollary 3.4, $P=P\left(d_{0}\right)$ where $d_{0} \in B^{1}(R, \mathbf{R})$. By Proposition 3.9, $d \in B^{1}(R, \mathbf{R})$. Thus there is a real-valued Borel function $g$ on $X$ such that $n=d\left(x, \tau^{n}(x)\right)=$ $g\left(\tau^{n}(x)\right)-g(x)$ a.e.; equivalently, $g\left(\tau^{n}(x)\right)=g(x)+n$. Consequently each of the sets $g^{-1}(r, \infty), r \in \mathbf{R}$, is invariant under $\tau$. It follows that unless $R$, and therefore, $M$, is type $\mathrm{I}$, each of these sets will be invariant under $\tau^{-1}$, too. In this event each $g^{-1}(r, \infty)$ is null or conull. Since this is absurd, we conclude that $M$ is type $\mathrm{I}$.

We believe that if $\mathfrak{P}=\mathfrak{P}(a)$, then $M$ is type I without the a priori assumption that $M$ is hyperfinite. The best we are able to prove, however, is the assertion that if $\mathfrak{P}=\mathfrak{P}(a)$, then $H^{1}(R, \mathbf{R})=0$ where $H^{1}(R, \mathbf{R})=Z^{1}(R, \mathbf{R}) / B^{1}(R, \mathbf{R})$. Of course, if $\mathfrak{P}=\mathfrak{P}(a)$, then Theorem 4.9 coupled with Proposition 3.9 implies that a $d \in Z^{1}(R, \mathbf{R})$ such that $d^{-1}(0)=\Delta$ must be a coboundary. The problem of handling the general cocycle is solved by the following theorem which is of independent interest. In Proposition 7.4 of [FM1], Feldman and Moore show that $R$ is type $I$ if and only if $H^{1}(R, G)=0$ for all abelian polonais groups $G$. It apparently is unknown if the vanishing of $H^{1}(R, \mathbf{R})$ implies that $R$ is type $\mathrm{I}$. 
The following theorem can be dug out of [LS] and [S2], but we give a self-contained proof fashioned on the theory we are developing. Larson and the third author show in [LS] that whenever $\mathfrak{A}$ is a nest subalgebra of a $\sigma$-finite von Neumann algebra $M$ and $\mathfrak{B}$ is a $\sigma$-weakly closed subalgebra of $M$ that contains $\mathfrak{A}$, then $\mathfrak{B}$ is a nest subalgebra of $M$. On the other hand, the third author shows in Theorem 2.19 of [S2] that under the same circumstances, if $\mathfrak{A}$ is an analytic subalgebra of $M$, then so is $\mathfrak{B}$.

THEOREM 4.10. Let $P \in \mathfrak{P}$ and let $Q$ be a transitive relation with $P \subseteq Q$. Then

(1) If $\mathfrak{T}(P)$ is an analytic subalgebra of $M$, so is $\mathfrak{T}(Q)$.

(2) If $\mathfrak{T}(P)$ is a nest algebra, so is $\mathfrak{T}(Q)$.

Proof. Suppose that $P=P(d)$ for some $d \in Z^{1}(R, \mathbf{R})$. We need to find a $d_{0} \in Z^{1}(R, \mathbf{R})$ so that $Q=P\left(d_{0}\right)$. Define a Borel function $g$ on $X$ as follows. Set $g(x)=\sup \{d(z, y) \mid(x, z),(x, y) \in Q \cap \theta(Q)$ and $(z, y) \in P\}$ provided the supremum is finite; otherwise, set $g(x)=1$. For $(x, y) \in P$, let $d_{1}(x, y)=\inf (d(z, w) \mid(z, x),(y, w) \in Q \cap \theta(Q)\}$ and let

$$
\tilde{d}_{0}(x, y)= \begin{cases}d_{1}(x, y)+\frac{1}{2}(g(x)+g(y)), & \text { if }(x, y) \notin Q \cap \theta(Q), \\ 0, & \text { if }(x, y) \in Q \cap \theta(Q) .\end{cases}
$$

Finally, define $d_{0}$ on $R$ by letting $d_{0}=\tilde{d}_{0}$ on $P$ and letting $d_{0}=$ $-\tilde{d}_{0} \circ \theta$ on $\theta(P)$. Note that since $\tilde{d}_{0} \mid \Delta=0, d_{0}$ is well defined on $R$. To show that $d_{0}$ is a cocycle, it suffices to show that for $(x, y)$ and $(y, z)$ in $P$,

$$
d_{0}(x, z)=d_{0}(x, y)+d_{0}(y, z) .
$$

So fix $(x, y)$ and $(y, z)$ in $P$. If $(x, y) \in Q \cap \theta(Q)$, then by definition, $d_{0}(x, y)=0, d_{1}(x, z)=d_{1}(y, z)$, and $g(x)=g(y)$. Hence, in this case, $d_{0}(x, z)=d_{0}(y, z)$, verifying (4.2). A similar argument verifies (4.2) when $(y, z) \in Q \cap \theta(Q)$. Thus we may assume that neither $(x, y)$ nor $(y, z)$ lies in $Q \cap \theta(Q)$. But then $(x, z) \notin Q \cap \theta(Q)$ and we have

$$
d_{0}(x, z)=d_{1}(x, z)+\frac{1}{2}(g(x)+g(z))
$$

and

$$
\begin{aligned}
d_{0}(x, y)+d_{0}(y, z)= & d_{1}(x, y)+d_{1}(y, z) \\
& +\frac{1}{2}(g(x)+2 g(y)+g(z)) .
\end{aligned}
$$

So, we need only prove that

$$
d_{1}(x, z)=d_{1}(x, y)+d_{1}(y, z)+g(y) .
$$


If $\sup \left\{d\left(w_{1}, w_{2}\right) \mid\left(y, w_{1}\right),\left(y, w_{2}\right) \in Q \cap \theta(Q)\right.$ and $\left.\left(w_{1}, w_{2}\right) \in P\right\}$ is infinite, then either there is a $w_{2} \in R(y)$ such that $d\left(y, w_{2}\right)>d(y, z)$ $(\geq 0)$ and $\left(y, w_{2}\right) \in Q \cap \theta(Q)$ or there is a $w_{1} \in R(y)$ such that $d\left(w_{1}, y\right)>d(x, y)(\geq 0)$. In the first instance, $(y, z)=\left(y, w_{2}\right) \circ\left(w_{2}, z\right)$ lies in $[\theta(Q) \circ \theta(Q)] \cap P \subseteq \theta(Q) \cap P \subseteq \theta(Q) \cap Q$, while in the second, we see by a similar calculation that $(x, y) \in Q \cap \theta(Q)$. Since we have already handled both of these cases before, they may be excluded and we may assume that the supremum is finite. So, by definition, we have

$$
g(y)=\sup \left\{d\left(w_{1}, w_{2}\right) \mid\left(y, w_{1}\right),\left(y, w_{2}\right) \in Q \cap \theta(Q),\left(w_{1}, w_{2}\right) \in P\right\} .
$$

Now fix $\varepsilon>0$ and $w_{1}, w_{2} \in X$ such that $\left(x, w_{1}\right)$ and $\left(w_{2}, z\right)$ lie in $Q \cap \theta(Q)$. We may then find $y_{1}, y_{2} \in X$ with $\left(y_{1}, y\right),\left(y_{2}, y\right) \in Q \cap \theta(Q)$ and $g(y) \leq d\left(y_{1}, y_{2}\right)+\varepsilon$. We then also have

$$
\begin{aligned}
& d_{1}(x, y)+d_{1}(y, z)+g(y) \\
& \quad \leq d\left(w_{1}, y_{1}\right)+d\left(y_{2}, w_{2}\right)+d\left(y_{1}, y_{2}\right)+\varepsilon=d\left(w_{1}, w_{1}\right)+\varepsilon .
\end{aligned}
$$

Since the choices of $\varepsilon>0$ and $w_{1}, w_{2}$ were arbitrary, subject only to the condition that $\left(x, w_{1}\right),\left(w_{2}, z\right) \in Q \cap \theta(Q)$, we conclude that

$$
d_{1}(x, y)+d_{1}(y, z)+g(y) \leq d_{1}(x, z) .
$$

For the reverse inequality, fix $\varepsilon>0$. Then there are $x_{1}, y_{1}, y_{2}$, and $z_{2}$ in $X$ such that $\left(x, x_{1}\right),\left(y, y_{1}\right),\left(y, y_{2}\right)$, and $\left(z, z_{2}\right)$ are all in $Q \cap$ $\theta(Q), d_{1}(x, y) \geq d\left(x_{1}, y_{1}\right)-\varepsilon, d_{1}(y, z) \geq d\left(y_{2}, z_{2}\right)-\varepsilon$, and $g(y) \geq$ $d\left(y, y_{2}\right)$. Hence

$$
\begin{aligned}
d_{1}(x, y)+d_{1}(y, z)+g(y) & \geq d\left(x_{1}, y_{1}\right)+d\left(y_{2}, z_{2}\right)+\left(y_{1}, y_{2}\right)-2 \varepsilon \\
& =d\left(x, z_{2}\right)-2 \varepsilon \geq d(x, z)-2 \varepsilon .
\end{aligned}
$$

Since $\varepsilon>0$ is arbitrary, equation (4.3) is satisfied and $d_{0}$ lies in $Z^{1}(R, \mathbf{R})$.

To complete the proof of (1), we need to show that $Q=\{(x, y) \in$ $\left.R \mid d_{0}(x, y) \geq 0\right\}$. Observe that since $(Q \cap \theta(Q)) \circ(Q \backslash \theta(Q)) \circ(Q \cap$ $\theta(Q)) \subseteq Q \backslash \theta(Q), d_{1}$ must be nonnegative on $Q \backslash \theta(Q)$, and, since $g \geq$ $0, d_{0}$ must be nonnegative on $Q \backslash \theta(Q)=P \backslash(Q \cap \theta(Q))$. Since $d_{0}$ is skew symmetric, $d_{0} \leq 0$ on $\theta(P) \backslash(Q \cap \theta(Q))$, and by definition, $d_{0}$ vanishes on $Q \cap \theta(Q)$. Recalling that $Q=P \cup(Q \cap \theta(Q))$, because $Q \backslash P \subseteq Q \cap \theta(P) \subseteq \theta(Q) \cap Q$, we see that $Q$ is contained in $\{(x, y) \in$ $\left.R \mid d_{0}(x, y) \geq 0\right\}$ which, in turn, is contained in $\left\{(x, y) \in R \mid d_{0}(x, y)=\right.$ $0\} \cup(Q \backslash \theta(Q))$. Thus, to finish, all we need to show is that $B:=$ $\left\{(x, y) \mid d_{0}(x, y)=0\right\} \backslash(Q \cap \theta(Q))$ is empty. But if $(x, y) \in B$, then $d_{1}(x, y)=g(x)=g(y)=0$ and using the definition of $g$, we see that $\{z \mid(z, x) \in Q \cap \theta(Q)\}=\{x\}$ while $\{w \mid(w, y) \in Q \cap \theta(Q)\}=\{y\}$. 
Consequently, $d_{1}(x, y)=d(x, y)=0$. Since this implies that $B \subseteq \Delta \subseteq$ $Q$, we see that $B$ is empty and that the proof of (1) is complete.

For (2), note that if $P \in \mathfrak{P}(n)$, then we may assume that $P=P(d)$ where $|d(x, y)|<1$ a.e. $\nu$. Then by the definition of $d_{0}$, we see that $\left|d_{0}(x, y)\right|<2$. Hence by Theorem 6 of [FM2], $d_{0}$ is a coboundary and $\mathfrak{T}(Q)$ is a nest subalgebra of $M$.

We note in passing that one can give a direct proof of part (2) of the preceding theorem along the following lines. Suppose that $g$ is a realvalued Borel function on $X$ such that $P=\{(x, y) \in R \mid g(y) \geq g(x)\}$ and assume, as we may, that $|g|<1$. If for each $x \in X$ we set $G(x)=$ $\{g(y) \mid(x, y) \in Q \cap \theta(Q)\}$ and if we set $g_{0}(x)=\sup G(x)+\inf G(x)$, then it is not difficult to see that $Q=\left\{(x, y) \in R \mid g_{0}(x) \leq g_{0}(y)\right\}$.

Corollary 4.11. If $\mathfrak{P}=\mathfrak{P}(a)$, then $H^{1}(R, \mathbf{R})=0$.

Proof. As remarked after Theorem 4.9, Theorem 4.9 coupled with Proposition 3.9 show that each $d \in Z^{1}(R, \mathbf{R})$ with $d^{-1}(0)=\Delta$ must be a coboundary. So let $d \in Z^{1}(R, \mathbf{R})$ be arbitrary and recall that since $X$ is standard, there is a Borel isomorphism $f$ from $X$ onto a subset of R. Let $d_{0}(x, y)=f(y)-f(x)$. Then $d_{0} \in Z^{1}(R, \mathbf{R})$ and $\Delta=d_{0}^{-1}(0)$. Let $P=\left\{(x, y) \mid\right.$ either $d_{0}(x, y) \geq 0$ and $d(x, y) \geq 0$ or $d_{0}(x, y) \leq 0$ and $d(x, y)>0\}$. Then it is easy to check that $P \in \mathfrak{P}$ and $P \subseteq P(d)$. Since $P \in \mathfrak{P}=\mathfrak{P}(n)$ (by Theorem 4.9), Theorem 4.10 implies that $P(d) \in \mathfrak{P}(n)$. By Proposition 3.9, $d$ is a coboundary.

5. The order induced by $P$. Throughout this section, $P$ will denote a fixed element of $\mathfrak{P}$. As we have pointed out before, $P$ induces a total ordering on each equivalence class. If $(y, x) \in R$, we shall often write $x \geq y$ if $(y, x) \in P$ and we shall write $x>y$ if $(y, x) \in P \backslash \Delta$. In [MSS] we randomized the analysis of total orders made in [R] in an effort to understand what possibilities there are for the order types of the equivalence classes $R(x), x \in X$. Our objective here is to show that if $P \in \mathfrak{P}(a)$, then the order type of each $R(x)$ must be quite limited. We must recall a certain amount of technical information from [MSS]. We present only the definitions and a little discussion; the reader should consult [MSS] for details.

Let $\Lambda$ denote the collection of all ordinals with the usual order, and let $\Lambda_{0}$ be the set of all countable ordinals. For each $\beta \in \Lambda$, we define a function $C_{\beta}$ from $R$ to $\mathbf{Z} \cup\{ \pm \infty\}$ by transfinite induction. For $\beta=0$ 
we set

$$
C_{0}(x, y)= \begin{cases}0, & \text { if } x=y, \\ \infty, & \text { if }(x, y) \in P \backslash \Delta, \\ -\infty, & \text { if }(x, y) \notin P .\end{cases}
$$

If $C_{\beta}$ has been defined and if $x \in X$, set $C^{\beta}(x)=\{y \in X \mid(x, y) \in$ $\left.R,\left|C_{\beta}(x, y)\right|<\infty\right\}$. Then define $C_{\beta+1}$ by the formula

$$
\begin{aligned}
C_{\beta+1}(x, y)= & \left|\left\{C^{\beta}(z) \mid x \leq z<y, C^{\beta}(z) \neq C^{\beta}(y)\right\}\right| \\
& -\left|\left\{C^{\beta}(z) \mid y \leq z<x, C^{\beta}(z) \neq C^{\beta}(x)\right\}\right|,
\end{aligned}
$$

where for a set $A,|A|$ denotes its cardinality. Note that because $P \in \mathfrak{P}$, one of the terms in the definition of $C_{\beta+1}$ must be zero. Thus the possibility that $C_{\beta+1}(x, y)$ is of the indeterminate form $\infty-\infty$ never arises, and $C_{\beta+1}$ is well defined. Finally, if $\gamma$ is a limit ordinal and if $C_{\beta}$ has been defined for all $\beta<\gamma$, then $C_{\gamma}$ is defined by the formula

$$
C_{\gamma}(x, y)= \begin{cases}\infty, & \text { if } C_{\beta}(x, y)=\infty \text { for all } \beta<\gamma \\ -\infty, & \text { if } C_{\beta}(x, y)=-\infty \text { for all } \beta<\gamma \\ 0, & \text { otherwise. }\end{cases}
$$

Each of the maps $C_{\gamma}$ is Borel by Lemma 5.1 of [MSS]. Note that $C^{0}(x)=\{x\}$ for every $x \in X$, so $C_{1}(x, y)=|\{z \mid x \leq z<y\}|-\mid\{z \mid y \leq$ $z<x\} \mid$. As a result, $C^{1}(x)=\{y \mid[x, y]$ is finite, if $y \geq x\} \cup\{y \mid[y, x]$ is finite, if $y \leq x\}$. It is clear that $C^{1}$ determines a condensation on each equivalence class of $X$, in the sense of $[\mathbf{R}]$ and that the $C^{\beta}$ 's, $\beta \in \Lambda$, also determine condensations on the equivalence classes of $X$-the $\beta$-fold iterates of the condensations determined by $C^{1}$ in the sense of [R, Definition 5.6]. Roughly speaking, a condensation is a homomorphism of a totally ordered set obtained by collapsing order intervals to points. The condensation $C^{1}$ collapses each finite order interval in each $R(x)$ to a point. Thus if the iterates of $C^{1}$ stabilize after a certain point, the image is either a single point or a densely ordered set. We define an analogue of the $F$-rank of a totally ordered set as follows. For $x_{0} \in X$, let $r\left(x_{0}\right)$ be the ordinal of the set $\{\alpha \in$ $\Lambda \mid C^{\alpha+1}(x) \neq C^{\alpha}(x)$ for some $\left.x \sim x_{0}\right\}$. Equivalently, $r\left(x_{0}\right)$ is the minimal ordinal $\beta$ satisfying $C^{\gamma}(x)=C^{\beta}(x)$ for all $\gamma \geq \beta$ and all $x \in$ $R\left(x_{0}\right)$. Observe that if $\beta$ is an ordinal such that $C^{\beta+1}(x)$ is different from $C^{\beta}(x)$ for some $x \in R\left(x_{0}\right)$, then there is a $y \in R\left(x_{0}\right)$ with $C_{\beta}(x, y)=\infty$ and $C_{\beta+1}(x, y)$ finite. It results, therefore, that there is an injective map from $\left\{\alpha \mid C^{\alpha}(x) \neq C^{\alpha+1}(x)\right.$ for some $\left.x \in R\left(x_{0}\right)\right\}$ into $R\left(x_{0}\right) \times R\left(x_{0}\right)$. Hence $r(x) \in \Lambda_{0}$ for every $x \in X$. Each of the 
sets $r^{-1}(\beta)$ is an invariant Borel set in $X$ by Lemma 5.2 of [MSS]. We say that $P$ is of type $D_{\alpha}, \alpha \in \Lambda_{0}$, if $r(x)=\alpha$ for almost every $x$. If there is an ordinal $\alpha \in \Lambda_{0}$ such that $r(x) \leq \alpha$ for almost all $x \in X$, then $X$ is the countable union of the disjoint, invariant, Borel subsets, $r^{-1}(\beta), \beta \leq \alpha$. It follows that if $R$ is ergodic and if there is some $\alpha \in \Lambda_{0}$ that majorizes $r(x)$ for almost all $x \in X$, then $P$ is of type $D_{\beta}$ for some $\beta \in \Lambda_{0}$. We suspect, but are unable to prove, that given $P$, with $R$ ergodic, there is a $\beta \in \Lambda_{0}$ that majorizes $r(x)$ for all $x$ in $X$. However, this point will not confront us here.

Our objective is the proof of the following theorem which shows that if $P \in \mathfrak{P}(a)$, and if $R$ is hyperfinite, then $P$ is of type $D_{0}$ or $D_{1}$. The example we gave in $\S 4$, Example 4.8 , is easily seen to be of type $D_{2}$. In any case, it will follow that it is quite easy to construct $P$ 's in $\mathfrak{P}$ that are not given by cocycles; i.e, $\mathfrak{P}(a) \neq \mathfrak{P}$.

TheOREM 5.1. Suppose that $R$ is finite and hyperfinite and let $P \in \mathfrak{P}$ be given by an $\mathbf{R}$-valued cocycle $d$ on $R$; i.e., suppose that $P=\{(x, y) \in$ $R \mid d(x, y) \geq 0\}$. Then $P$ is of type $D_{0}$ or $D_{1}$.

Proof. Without loss of generality, we may assume that $R$ is given by an ergodic measure preserving transformation, say $\tau: R=\left\{\left(x, \tau^{n} x\right) \mid\right.$ $n \in \mathbf{Z}, x \in X\}$. If $d$ is a transient cocycle, then for almost all $(x, y) \in$ $R,\left|C_{1}(x, y)\right|<\infty$ by Corollary 11.3 of [Sc]. Hence, in this case, $P$ is in $D_{1}$. On the other hand, if $d$ is recurrent then, for almost all $x \in X, C^{1}(x)$ has either one or two elements (i.e., almost every $R(x)$ is densely ordered or densely ordered "with some gaps"). To see this, suppose not and choose two points $(x, y)$ and $(y, z) \in P$ with $C_{1}(x, y)=C_{1}(y, z)=1$. Set $\varepsilon=\min (d(x, y), d(y, z))(>0)$, set $x=\tau^{n}(y), z=\tau^{k}(y)$, and set $N=\max (|n|,|k|)$. Then for each integer $l$ with $|l|>N$, we have $\left|d\left(x, \tau^{l}(x)\right)\right|>\varepsilon$, which contradicts the recurrence of $d$ by Corollary 11.2 of [Sc]. Hence $\left|C^{1}(x)\right| \leq 2$, a.e. so that $P$ is of type $D_{0}$ or $D_{1}$.

COROLlARY 5.2. If $\gamma$ is a countable ordinal greater than one, if $G=\mathbf{Z}^{\gamma}$, with the lexicographic order, acts freely and ergodically on the standard Borel space $X$, leaving invariant a probability measure, and if $P=\{(x, g \cdot x) \mid x \in X, g \geq 0\}$, then $P \notin \mathfrak{P}(a)$.

Proof. Since $R_{G}$ is finite and hyperfinite, and since $P$ is of type $D_{\gamma}$, by Lemma 5.4 of [MSS], the result is immediate from Theorem 5.1. 
REMARK 5.3. One might think, on the basis of Theorem 1 of [FM1], that given $R$ and $P$, it ought to be possible to find a totally ordered group $G$ with positive semigroup $\Sigma$ such that $R=R_{G}$ and $P=\left\{(x, g \cdot x) \in R_{G} \mid g \in \Sigma\right\}$. However, a moment's reflection reveals that this is not possible. Simply consider the usual ordering on the finite set $\{1,2, \ldots, n\}$. On the other hand, there is a cocycle $c$ on $R$ with values in a totally ordered group, namely $\mathbf{R}$, such that this $P$ is $\{(x, y) \mid c(x, y) \geq 0\}$. It is natural to ask, therefore, whether a given $P$ is of this form; i.e. when does there exist a group $G$ with positive semigroup $\Sigma$ and a cocycle $c: R \rightarrow G$ such that $P=\{(x, y) \in R \mid c(x, y) \in \Sigma\}$ ? We suspect, on the basis of our analysis in [MSS], that if $P$ is of type $D_{\alpha}$, then it is possible to realize $P$ as $\{(x, y) \in R \mid c(x, y) \in \Sigma\}$ where $G$ is either $\mathbf{Z}^{\alpha}$ or $\mathbf{Z}^{\alpha} \mathbf{R}$ and these groups are given the lexicographical ordering. We intend to investigate this in the future.

\section{REFERENCES}

[A1] Wm. B. Arveson, Analyticity in operator algebras, Amer. J. Math., 89 (1967), 578-642.

[A2] - On groups of automorphisms of operator algebras, J. Funct. Anal., 15 (1974), 217-243.

[A3] Operator algebras and invariant subspaces, Annals of Math., 100 (1974), 433-532.

[AFG] E. Azoff, C. Fong, and F. Gilfeather, A reduction theory for non-self-adjoint operator algebras, Trans. Amer. Math. Soc., 224 (1976), 351-366.

[FM1] J. Feldman and C. Moore, Ergodic equivalence relations, cohomology and von Neumann algebras. I, Trans. Amer. Math. Soc., 234 (1977), 289-324.

[FM2] Ergodic equivalence relations, cohomology, and von Neumann algebras. II, Trans. Amer. Math. Soc., 234 (1977), 325-359.

[GL] F. Gilfeather and D. Larson, Nest-subalgebras of von Neumann algebras, Advances in Math., 46 (1982), 171-199.

[KS] R. Kadison and I. Singer, Triangular operator algebras, Amer. J. Math., 82 (1960), 227-259.

[LS] D. Larson and B. Solel, Nests and inner flows, J. Operator Theory, 16 (1986), 157-164.

[LM] R. Loebl and P. Muhly, Analyticity and flows in von Neumann algebras, J. Funct. Anal., 29 (1978), 214-252.

[M] R. Mercer, Bimodules over Cartan subalgebras, Rocky Mountain J. Math., (to appear).

[MSS] P. S. Muhly, K. Saito and B. Solel, Coordinates for triangular operator algebras, Ann. of Math., 127 (1988), 245-278.

[R] J. Rosenstein, Linear Orderings, Academic Press, New York, 1982.

[Sc] K. Schmidt, Lectures on Cocycles of Ergodic Transformation Groups, Macmillan, Bombay, 1977. 
[S1] B. Solel, Analytic operator algebras (factorization and expectation), Trans. Amer. Math. Soc., 287 (1985), 799-818.

[S2] $\frac{}{63-89}$. Maximality of analytic operator algebras, Israel J. Math., 62 (1988),

Received February 2, 1988. The first author was supported in part by the National Science Foundation. The second author was supported in part by a Grant-in-Aid for Scientific Research from the Japanese Ministry of Education.

THE UNIVERSITY OF IOWA IOWA CITY, IA 52242

NiIGATA UNIVERSITY

IKARASHI 2-8050, NIIGATA

950-21 JAPAN

AND

UNIVERSITY OF HAIFA

HAIFA 31999, ISRAEL 



\section{PACIFIC JOURNAL OF MATHEMATICS EDITORS}

\author{
V. S. VARADARAJAN \\ (Managing Editor) \\ University of California \\ Los Angeles, CA 90024 \\ Herbert Clemens \\ University of Utah \\ Salt Lake City, UT 84112 \\ THOMAS ENRIGHT \\ University of California, San Diego \\ La Jolla, CA 92093
}

R. FINN

Stanford University

Stanford, CA 94305

HeRmann FlaschKa

University of Arizona

Tucson, AZ 85721

VAUGHAN F. R. Jones

University of California

Berkeley, CA 94720

STEVEN KeRCKHOFF

Stanford University

Stanford, CA 94305
ROBION KIRBY

University of California

Berkeley, CA 94720

C. C. MOORE

University of California

Berkeley, CA 94720

HAROLD STARK

University of California, San Diego

La Jolla, CA 92093

\section{ASSOCIATE EDITORS}
R. ARENS
E. F. BECKENBACH
B. H. NeUMANN
F. WolF
K. YoshidA (1906-1982)

\section{SUPPORTING INSTITUTIONS}

UNIVERSITY OF ARIZONA

UNIVERSITY OF BRITISH COLUMBIA

CALIFORNIA INSTITUTE OF TECHNOLOGY

UNIVERSITY OF CALIFORNIA

MONTANA STATE UNIVERSITY

UNIVERSITY OF NEVADA, RENO

NEW MEXICO STATE UNIVERSITY

OREGON STATE UNIVERSITY

\author{
UNIVERSITY OF OREGON \\ UNIVERSITY OF SOUTHERN CALIFORNIA \\ STANFORD UNIVERSITY \\ UNIVERSITY OF HAWAII \\ UNIVERSITY OF TOKYO \\ UNIVERSITY OF UTAH \\ WASHINGTON STATE UNIVERSITY \\ UNIVERSITY OF WASHINGTON
}

The Supporting Institutions listed above contribute to the cost of publication of this Journal, but they are not owners or publishers and have no responsibility for its content or policies.

Mathematical papers intended for publication in the Pacific Journal of Mathematics should be in typed form or offset-reproduced (not dittoed), double spaced with large margins. Please do not use built up fractions in the text of the manuscript. However, you may use them in the displayed equations. Underline Greek letters in red, German in green, and script in blue. The first paragraph must be capable of being used separately as a synopsis of the entire paper. In particular it should contain no bibliographic references. Please propose a heading for the odd numbered pages of less than 35 characters. Manuscripts, in triplicate, may be sent to any one of the editors. Please classify according to the scheme of Math. Reviews, Index to Vol. 39. Supply name and address of author to whom proofs should be sent. All other communications should be addressed to the managing editor, or Elaine Barth, University of California, Los Angeles, California 90024.

There are page-charges associated with articles appearing in the Pacific Journal of Mathematics. These charges are expected to be paid by the author's University, Government Agency or Company. If the author or authors do not have access to such Institutional support these charges are waived. Single authors will receive 50 free reprints; joint authors will receive a total of 100 free reprints. Additional copies may be obtained at cost in multiples of 50 .

The Pacific Journal of Mathematics is issued monthly as of January 1966. Regular subscription rate: $\$ 190.00$ a year (5 Vols., 10 issues). Special rate: $\$ 95.00$ a year to individual members of supporting institutions.

Subscriptions, orders for numbers issued in the last three calendar years, and changes of address should be sent to Pacific Journal of Mathematics, P.O. Box 969, Carmel Valley, CA 93924, U.S.A. Old back numbers obtainable from Kraus Periodicals Co., Route 100, Millwood, NY 10546.

The Pacific Journal of Mathematics at P.O. Box 969, Carmel Valley, CA 93924 (ISSN 0030-8730) publishes 5 volumes per year. Application to mail at Second-class postage rates is pending at Carmel Valley, California, and additional mailing offices. Postmaster: send address changes to Pacific Journal of Mathematics, P.O. Box 969, Carmel Valley, CA 93924.

\section{PUBLISHED BY PACIFIC JOURNAL OF MATHEMATICS, A NON-PROFIT CORPORATION}




\section{Pacific Journal of Mathematics}

Vol. 137, No. $2 \quad$ February, 1989

Alain Connes and E. J. Woods, Hyperfinite von Neumann algebras and Poisson boundaries of time dependent random walks ............ 225

R. Coquereaux and D. Kastler, Remarks on the differential envelopes of

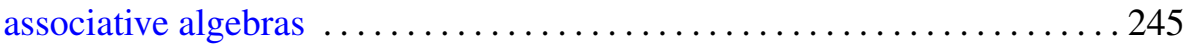

Uffe Haagerup, The injective factors of type $\mathrm{III}_{\lambda}, 0<\lambda<1 \ldots \ldots \ldots 265$

Vaughan Jones, On knot invariants related to some statistical mechanical

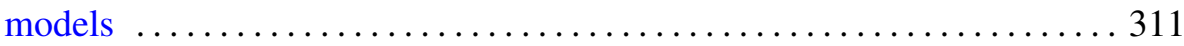

Paul Scott Muhly, Kichi-Suke Saito and Baruch Solel, Coordinates for triangular operator algebras. II ......................... 335

Klaus Schmidt, Mixing automorphisms of compact groups and a theorem by Kurt Mahler ........................................ 371

Irving E. Segal, Algebraic characterization of the vacuum for quantized

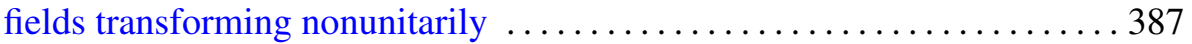

Colin Eric Sutherland and Masamichi Takesaki, Actions of discrete amenable groups on injective factors of type $\mathrm{III}_{\lambda}, \lambda \neq 1 \ldots \ldots \ldots \ldots 405$ 